Historic, Archive Document

Do not assume content reflects current scientific knowledge, policies, or practices. 

SPECIAL LIST NO. 79

\title{
ESTABLISHED ORCHIDS
}

\section{AND NATIVE HARDY ORCHIDS}

THIS LIST CANCELS ALL PREVIOUS ISSUES

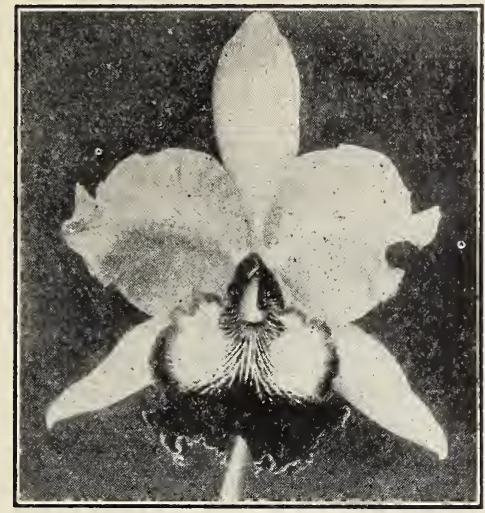

JOHN E. LAGER

HENRY HURRELL

\section{LAGER \& HURRELL Orchid Growers and Importers} SUMMIT, NEW JERSEY

CABLE ADDRESS

"ABBACHIO" SUMMITNEWJERSEY.

A. B. C. CODE

4 TH AND 5TH EDITIONS USED

\author{
LOCAL AND LONG \\ DISTANCE TELEPHONE \\ $438 \mathrm{~W}$ SUMMIT
}

\section{Dear Sir:-}

We herewith take pleasure in handing you special list No. 79, in which you will find as large and varied a collection of Orchids as it is possible to find anywhere. You will also find listed some of our native hardy orchids but these we can only furnish in the spring and Fall.

\section{ALL PLANTS MARKED $\times$ ARE HYBRIDS.}

In ordering please use number in left margin only and give number of list.

All prices in this list are net cash, F. O. B. Summit, N. J., but $2 \%$ may be deducted when remittance is sent with.order.

Trusting to be favored with your valued order.

We are, yours respectfully,

LAGER \& HURRELL. 


\section{ORCHIDS, CULTURAL DIRECTIONS}

The orchids are without question the most beautiful and most lasting flowers in all the floral kingdom, and also the easiest grown plants of any, if a few common-sense points are observed.

Ventilation-Orchids delight in fresh air at all times when weather permits. During the warmest part of the summer months it is beneficial to leave the ventilators slightly open at night.

Shading-The glass should be lightly shaded from about February 15 th to September 1st, or the sun will burn the plants. During the rest of the year clear glass is desirable.

Watering-When watering see that plenty is given and then allow the plants to dry out before giving more, this will keep the compost in the pots in good condition for a long time. The atmosphere in the house should be moderately moist.

Temperature-A, night temperature of 58 to 60 and correspondingly higher in the daytime will suit the greater number of orchids.

Potting-Firm potting is absolutely necessary. Orchids will not grow well and thrive if loose in the pots or baskets. In repotting, care should be taken not to disturb the roots, rather break the pots, while the baskets may be taken apart carefully and the plants placed in new baskets working the material around the plants firmly. This is generally done by placing the material used between the rim of the pot or basket, and the ball of the plant pressing the material towards the plant. When no more material can be conveniently used it will be found the plants are very firm in the receptacle. Avoid overpotting and do not disturb the plants unless necessary.

Materials for Potting-The best material for potting orchids is osmunda fibre, commonly called peat. A few lumps of charcoal and pieces of broken pots may be added to advantage, also a little live sphagnum moss.

Timo for Potting-In the greater majority of cases the time for potting is immediately after the plants finish flowering. The plants are more dormant than at any other other time notably wuch as Cattleyas, Laelias, Oncidiums, and Odontoglossums and many more. With these general directions anyone will be able to grow orchids and reap a rich harvest of these exquisite flowers. 


\section{Addenda to Special List No. 79}

Cymbidium Hybrids and species, mostly Hybrids, names lost; fine plants in 3 and 4 -inch pots, 1 strong shoot, each

Oncidium Cheirophorum, a pretty species, seldom seen, 5 to 6 bulbs, each.................................................. $\quad 5.00$

Oncidium Forbesii, 3 to 4 bulbs, each 5.00

Oncidium Marshallianum, 3 to 4 bulbs, each 5.00

Oncidium Marshallianum, 3 to 4 bulbs, stronger, each........ 7.50

Oncidium Varicosum Rogersii, 3-4 bulbs.......................... 5.00

Oncidium Varicosum Rogersii, 3-4 bulbs, stronger.............. 7.50

Zygopetalum Mackayii, a fine old orchid for cut flowers, 2 to 3 bulbs, each. 
1. Acampe Pappilosa, 16 leaves................................\$ 5.00

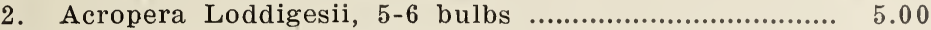

3. Acineta species, $3-5$ bulbs....................................... 5.00

4. Ada Aurantiaca , 4-5 bulbs..................................... 7.50

5. Aerides Affine, 8 leaves......................................... 10.00

6. Aerides Crassifolium, 7 leaves............................... 10.00

7. Aerides Crassifolium, 12 leaves.............................. 15.00

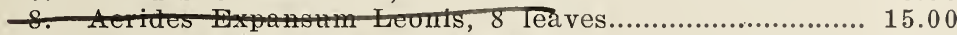

T. Arider Ierpentao, 10 leaves..................................... 15.00

10. Itawereae, 6 leaves................................. 20.00

11. Aorides Odoratum Majus, 7 leaves.n......................... 10.00

12. Aerides Odaratum Majus, 15 leaves.......................... 25.00

13. Aerides Vandarum, 1 stem 12 inches high................. 10.00

14. Aerides Species, 10 leaves...................................... 15.00

15. Aerides Species, 14 leaves..................................... 20.00

16. Aerides Quinquevulnerum, 5 leaves........................ 7.50

17. Angraecum Distichum, 6-8 stemis.......................... 10.00

18. Angraecum Eichlerianum, 15 leaves and more.......... 10.00

19. Angraecum Eichlerianum, specimens..........\$20.00 to 25.00

20. Angraecum Eburneum, 8 large leaves....................... 20.00

21. Angraecum Eburneum, 13 to 14 leaves....................... 35.00

22. Angraecum Sesquipedale, 6 leaves.......................... 15.00

23. Angraecum Sesquipedale, 8 leaves............................ 20.00

24. Angraecum Sesquipedale, 10 to 12 leaves.................. 40.00

25. Batemania Burtii, 1 shoot...................................... 15.00

26. Batemania Wallisii var. Major,1 shoot $x-x$ var....... 50.00

27. Bifrenaria Harrisoniae, 5 bulbs............................... 7.50

28. Brassavola Digbyana, 4 bulbs................................. 7.50

29. Brassia brachiata, $3-4$ bulbs.............................. 5.00

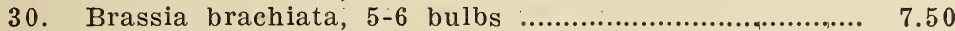

31. Brassia Lawrenceana Longissima, 4 bulbs................. 10.00

32. Brassia Verrucosa, 4-5 bulbs ................................. 5.00

33. Brassia Verrucosa, $6-8$ bulbs .................................. 7.50

\section{BRASSO-CATTLEYA}

These are the most.gorgeous and beautiful of all orchids produced by the Hybridizer's skill. Culture same as Cattleyas.

35. Brasso-Cattleya $\times$ Carmen, 4 bulbs........................ 35.00

36. Brasso-Cattleya $X$ Forbesil $X$ Digbyana, 5 bulbs....... 20.00

37. Brasso-Cattleya $\times$ Fournierii, 4 bulbs.......................2 25.00

38. Brasso-Cattleya $\times$ Fournierii, 5 buibs..................... 30.00

39. Brasso-Cattleya $\times$ Heatonense, 4 bulbs.................... 25.00

40 Brasso-Cattleya- $\times$ Holfordii, 5 bulbs ...................... 20.00

41. Brasso-Cattleya $\times$ Ilene, 4 bulbs................................. 35.00

42. Brasso-Cattleya $\times$ Ilene, 5 bulbs........................... 50.00

43. Brasso-Cattleya- $x$ Imperatrice de Russie, 3 bulbs..... 25.00

44. Brasso-Cattleya- $\times$ Jessopi- (Digbyana $\times$ Xanthina), 4 bulbs .......................................................... 25.00

45. Brasso-Cattleya $\times$ King Edward VII, 4 bulbs............. 30.00

46: Brasso-Cattleya $\times$ Leemanii, 4 bulbs............................. 35.00

47. Brasso-Cattleya $\times$ Maronae, 4 bulbs ........................ 25.0 250

48. Brasso-Cattleya $\times$ Maronae, 5 bulbs......................... 30.00 
49. Brasso-Cattleya $\times$ Maronae, gigantea, 4 bulbs

50. Brasso-Cattleya $\times$ Marie, 4 bulbs

51. Brasso-Cattleya $\times$ Mars, 3 bulbs.

25.00

52. Brasso-Cattleya $\times$ Mirabilis, 3 bulbs

-58 Brand

5. Brasso-Cattleya X. Oberon, 5 butbs -.............................

20.00

55. Brasso-Cattleya $\times$ Ruby, 3 bulbs

25.00

56. Brasso-Cattleya $\times$ Sedenii, 4 bulbs

25.00

57. Brasso-Cattleya $\times$ Speciosa, 3 bulbs

5.00

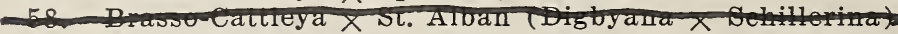

59. Brasso-Cattleya $\times$ Thorntonii, 4 bulbs.

15.00

60. Brasso-Cattleya $\times$ Thorntonii, 5 bulbs

25.00

61. Brasso-Cattleya $\times$ Veitchii, 4 bulbs

0.00

62. - Cat $x$.

64. Brasso-Cattleya $\times$ Victoria Regina, 4 bulbs..................

5.00

30.00

35.00

65. Brasso-Cattleya $\times$ Watteau, 4 bulbs .................. 30.00

66. Brasso-Cattleya $\times$ Wotan, 4 bulbs ............................. 50.00

67. Brasso-Laelia $\times$ Helen, 4 bulbs................................ 25.00

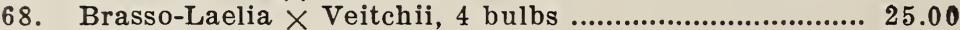

69. Brasso-Laelia Veitchii, 5 bulbs ............................ 30.00

70. Brasso-Laelia-Cattleya $\times$ Decia, 3 bulbs

71. Brasso-Laelia-Cattleva $\times$ Everest, var. Venus, one of the finest of all, 4 strong bulbs...

72. Brasso-Laelia-Cattleya $\times \mathrm{H}$. Hunter, 3 bulbs............ 25.00

73. Brasso-Laelia-Cattleya $\times$ Monitor, 3 bulbs...............2 25.00

74. Brasso-Laelia-Cattleya $\times$ Orient, 3 bulbs $\ldots \ldots \ldots \ldots \ldots \ldots \ldots . . \ldots 25.00$

75. Brasso-Laelia-Cattleya $\times$ Truffautiana, 3 bulbs...........2 25.00

76. Bulbophyllum Careyanum, 8-10 bulbs ..................... 5.00

77. Bulbophyllum Bearii, 5 bulbs ............................ 5.00

78. Bulbophyllum Grandiflorum, 5-6 bulbs .................. 5.00

79. Bulbophyllum Grandiflorum, 7-8 bulbs ................... 7.50

80. Bulbophyllum Grandiflorum, $9-10$ bulbs ................ 10.00

81. Bulbophyllum Lobbii, $6-8$ bulbs ................................ 5.00

82. Bulbophyllum Lobbii, $9-12$ bulbs............................. 7.50

83. Bulbophyllum Odoratissimum, 20-25 bulbs ............ 5.00

\section{CALANTHES}

The Calanthes are deciduous orchids and should be potted in Abrous loam, peat, leafsoil and sand with plenty of water during the growing season. The handsome sprays carry numerous flowers of rose pink to pure white, with crimson lips, according to kinds, and are valuable for cutting purposes. Flower during winter months.

84. Calanthe $\times$ Bella, 1 bulb $\$ 5.00$

85. Calanthe $\times$ Clive, 1 bulb

86. Calanthe $\times$ Harrisii, 1 bulb ................................. 5.00

87. Calanthe $\times$ Jobstown, pure white, 1 bulb................ 5.00

88. Calanthe $\times$ McWilliamsii, 1 bulb .......................... 5.00

89. Calanthe $\times$ Mrs. Cornelius Vanderbilt, 1 bulb........... 5.00

90. Calanthe Regnierii, 1 bulb ....................................... 3.50

91. Calanthe $\times$ Sandhurstiana, 1 bulb .......................... 5.00

92. Calanthe Veitchii, 1 bulb ..................................... 3.50 
93. Calanthe Veitchii Rosea, 1 bulb............................ 3.50

94. Calanthe $\times$ Summitense, 1 bulb ............................ 3.50

95. Calanthe veratrifolia, 1 shoot............................ 5.00

96. Camarotis purpurea, 1 stem ................................ 5.00

97. Camarotis purpurea, 2 stems ............................. 7.50

98. Camarotis Manii, 1 stem ...................................... 5.00

99. Camarotis Manii, 2 stems .................................... 7.50

100. Catasetum Maculatum, 2 bulbs .......................... 5.00

\section{C.ATTLEYAS}

These are the showiest of all the orchids and need no comment -whether used in the conservatory, or for exhibition purposes, or cut flowers. They have no equal. For culture see page 2.

101. Cattleya $\times$ Adula, 4 bulbs ....................................\$2 $\$ 200$

102. Cattleya \& Alfred Dimmock (Laurenceana $\times$ Aurea) 4 bulbs

103. Cattleya $\times$ Alex $(\mathrm{Tum}$ is $\times$ Aurea $), 4$ bulbs............... 50.00

104. Cattleya Amethystoglossa, 4 bulbs .......................... 10.0 00

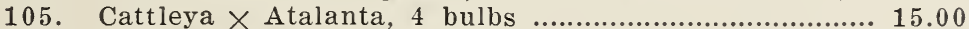

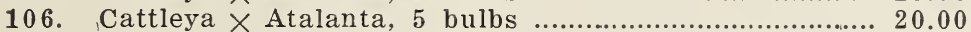

107. Cattleya $\times$ Amabilis, 4 bulbs ................................. 25.00

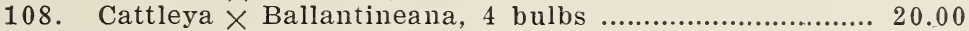

109. Cattleya Bowringeana, $5-6$ bulbs .......................... $\quad 7.50$

110. Cattleya Bowringeana, $7-8$ bulbs .......................... 10.00

111. Cattleya Bowringeana, 10-12 bulbs ..................... 15.00

112. Cattleya Bowringeana, specimens, 18-25 bulbs.........25.00

113. Cattleya Chrysotoxa, 4 bulbs ................................ 15.00

114. Cattleya Chrysotoxa, 5 bulbs ............................... 20.00

115. Cattleya Chrysotoxa, 6 bulbs ................................... 25.00

116. Cattleya $\times$ Clarissa, 5 bulbs .............................. 15.00

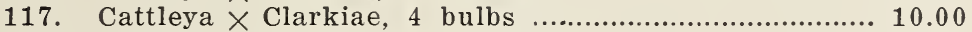

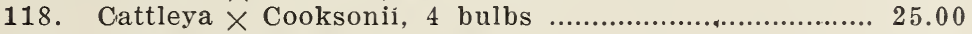

119. Cattleya Dowiana, 5 bulbs ..................................... 15.00

120. Cattleya Dowiana, 6 bulbs ........................................ 20.00

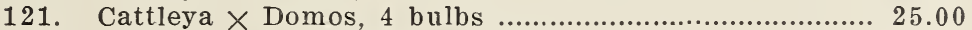

122. Cattleya $\times$ Dupreyana, 4 bulbs ............................ 25.00

123. Cattleya $\times$ Dusseldorfii, undine, 4 bulbs..................25.00

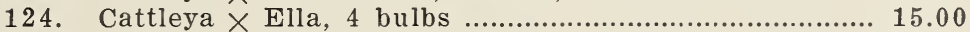

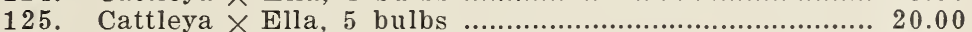

126. Cattleya Elongata, 4 bulbs ..................................... 15.00

127. Cattleya $\times$ Enid, 4 bulbs .................................. 20.00

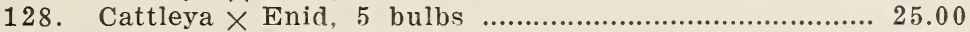

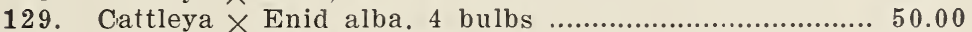

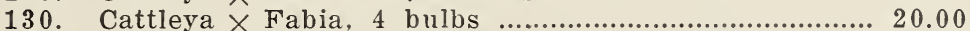

131. Cattleya $\times$ Fabia, 5 bulbs .................................... 25.0 0

132. Cattleya $\times$ Fabia alba, 4 bulbs.......................... 35.00

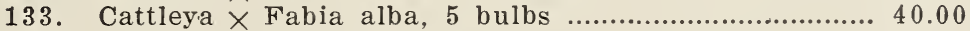

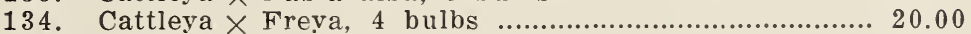

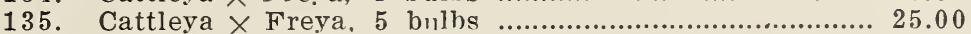

136. Cattleya Gaskelliana, $5-6$ bulbs ............................. 5.00

137. Cattleya Gaskelliana, $7-8$ bulhs.............................. 7.50

138. Cattleya Gaskelliana, $9-10$ bulbs......................... 10.00

139. Cattleya Gaskelliana, 11-12 bulhs ............................ 15.00

140. Cattleya Gaskelliana alba, 4 bulbs........................... 50.00 
141. Cattleya Gaskelliana Albescens, 6 bulbs................... 25.00

142. Cattleya Gaskelliana White sepals and petals, purple

lip, $\times \times$ fine variety, 4 bulbs........................... 50.00

143. Cattleya Gigas, 4-5 bulbs....................................... 10.00

144. Cattleya Gigas, $6-7$ bulbs...................................... 15.00

145. Cattleya Gigas, $8-9$ bulbs.................................... 20.00

146. Cattleya Gigas, $10-12$ bulbs................................... 30.00

147. Cattleya Gigas Alba (very rare). Price on application.

148. Cattleya Gigas Rochellense. Price on application.

149. Cattleya $\times$ Gildenii, 4 bulbs................................ 25.0 0

150, Catteya Granutosa, 4 butbs...................................... 10.00

151. Cattleya $\times$ Guttata $\times$ Hardyana, 4 bulbs..................... 20.0 200

152. Cattleya $\times$ Hardyana, 5 bulbs............................. 25.00

153. Cattleya $\times$ Hardyana, 6 bulbs.............................. 30.00

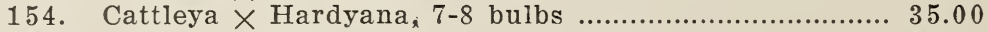

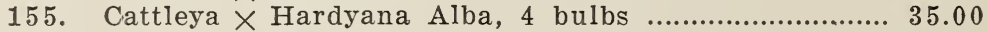

156. Cattleya $\times$ Hardyana Rosea, 5 bulbs....................... 35.00

157. Cattleya $\times$ Hardyana Springbrook Var., 4 bulbs......... 50.00

158. Cattleya $\times$ Hardyana $\times$ Leopoldii, 4 bulbs.............. 15.00

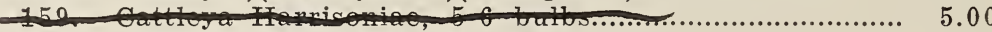

160. Cattleya Intermedia Alba, 4 bulbs .......................... 35.00

161. Cattleya $\times$ Iris, 5 bulbs........................................ 20.00

162. Cattleya Lawrenceana, 4-5 bulbs............................... 20.00

163. Cattleya Labiata, 5-6 bulbs................................... 10.00

164. Cattleya Labiata Rosea, 4 bulbs............................... 15.00

165. Cattleya Labiata, white sepals and petals, with blush rose lip, 4 bulbs.................................................. 35.00

166. Cattleya Labiata Cooksonii, 4 bulbs....................... 40.00

167. Cattleya $\times$ Leda, 4 bulbs........................................ 25.00

168. Cattleya $\times$ Lord Rotschild, 4 bulbs.......................25.00

169. Cattleya Lobata, 4 bulbs...................................... 10.00

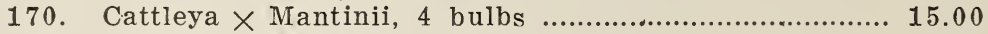

171. Cattleya $\times$ Mantinii, 5 bulbs.............................. 20.00

172. Cattleya $\times$ Minucea, $4-5$ bulbs.................................. 15.00

173. Cattleya Mendelii, 4-5 bulbs..................................... 10.00

174. Cattleya Mendelii, 6-7 bulbs...................................... 12.50

175. Cattleya Mendelii Columbia, 5 bulbs......................... 25.00

176. Cattleya Maxima, very scarce, 4 bulbs....................... 15.00

177. Cattleya $\times$ Maggie Raphael Alba, 4 bulbs.................. 35.00

178. Cattleya $\times$ Miss Williams, 5-6 bulbs........................ 10.00

179. Cattleya $\times$ Moira, 4 bulbs.....................................2 20.00

180. Cattleya Mossiae, 4-6 bulbs.................................. 10.00

181. Cattleya Mossiae, $7-8$ bulbs ....................................... 12.50

182. Cattleya Mossiae, $9-10$ bulbs..................................... 20.00

18\% Cona $1225 \mathrm{bulb}$ s 250050.00

184. Cattleya Mossiae Alba, 4 bulbs............................... 35.00

185. Cattleya Mossiae Grandiflora, 4-5 bulbs................... 25.00

186. Cattleya Mossiae Reineckiana, 4-5 bulbs...................2 25.00

187. Cattleya $\times$ Octavia, 4 bulbs.................................... 20.00

188. Cattleya $\times$ Octavia, 5 bulbs ................................... 25.00

189. Cattleya Percivaliana, $5-7$ bulbs................................ 5.00

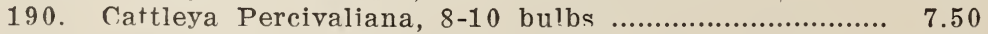

191. Cattleya Percivaliana, $11-12$ bulbs......................... 10.00

192. Cattleya Percivaliana, 13-15 bulbs .......................... 15.00 
193. Cattleya Percivaliana Alba, 5 bulbs

194. Cattleya Percivaliana Roeblingiana, (white sepals and petals, rose-purple lip) 4 bulbs...................... 35.00

195. Cattleya $\times$ Peter, 4 bulbs ...................................... 30.00

196. Cattleya $\times$ Peetersii, 4 bulbs ................................. 30.00

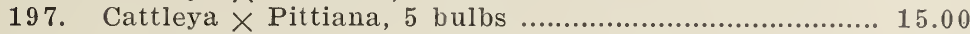

198. Cattleya $\times$ Portia, 4 bulbs.................................. 15.00

199. Cattleya $\times$ Portia, 5 bulbs.................................. 20.00

200. Cattleya Rex, on application.

201. Cattleya Schroederae, $4-5$ bulbs............................. 7.50

\begin{tabular}{|c|c|}
\hline 3 . & Cattleya Schroederae Citrina, 4 bulbs. \\
\hline & Cot+ogenele \\
\hline & erii, $4-5$ bulbs \\
\hline & Cattleya $\times$ Skinnerii, $6-7$ bulbs \\
\hline 207. & Cattleya $\times$ Soulange, 4 bulbs.. \\
\hline & Cattleya Speciossissima, 4-5 bul \\
\hline & avior, 5 bulbs... \\
\hline [0. & Cattleya X Pturgodian, 4 , \\
\hline 211. & Cattleya Trianae, 5-6 bulbs... \\
\hline 212. & Cattleya Trianae, 7-10 bulbs.. \\
\hline 213. & Cattleya Trianae, $11-12$ bulbs \\
\hline 14 & Cattleya Trianae, 13-15 bulbs.... \\
\hline 15. & 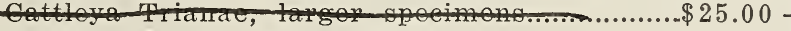 \\
\hline 216. & Cattleya Trianae Alba, 4 bulbs. \\
\hline 217. & Cattleya Trianae Amesiana, 5 bulbs... \\
\hline 218. & Cattleya Trianae Atropurpurea, 4-5 bulbs..... \\
\hline & 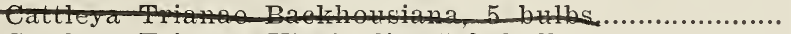 \\
\hline 2 & Cattleya Trianae Virginalis, 5-6 bulbs, \\
\hline & $\mathrm{Ca}$ \\
\hline & n................... \\
\hline
\end{tabular}

224. Cattleya $\times$ Zenia, 4 bulbs...................................... 20.0 00

225. Cattleya $\times$ Zephyr $($ Schroederae $\times$ Aurea), 4 bulbs... 35.00

\section{COELOGYNE}

226. Coelogyne Asperata, 5 bulbs

5.00

227. Coelogyne Asperata, 6-7 bulbs

7.50

228. Coelogyne Asperata specimens, 8-10 bulbs.

10.00

229

Coelogyne Barbata, 4-5 bulbs

5.00

230 .

Coelogyne Barbata, 6-7 bulbs.

7.50

231

Coelogyne Cristata, 5-6" pots, flowers white.

5.00

232. Coelogyne Cristata, 7-8" pots, flowers white.

15.00

233. Coelogyne Cristata Maxima, 5" pans, flowers white....

5.00

234. Coelogyne Cristata Alba, 5" pots, flowers white..........

7.50

235. Coelogyne Dayana, 5-6 bulbs....

5.00

236. Coelogyne Dayana, 7-8 bulbs....

7.50

237. Coelogyne Flaccida, 4-5 bulbs......................................

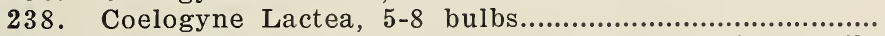

239. Coelogyne Lactea, 7-8 bulbs; a fine orchid, easily grown

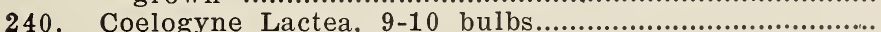


242. Coelogyne Massangeana, 7-8 bulbs......................... 7.50

243. Coelogyne Massangeana, 9-10 bulbs....................... 10.00

244. Coelogyne Oscellata Maxima, 5 bulbs...................... 5.00

245.. Coelogyne Pandurata -a very handscme orchid with large green flowers, and with a lip almost black. An easy, fast-growing plant; does well in a night temperature of $60^{\circ}$ and higher in the daytime. See cut below.

246. Coelogyne Panduraia, 2-3 small bulbs...................... 10.00

247. Coelogyne Pandurata, 3-4 bulbs.............................. 15.00

248. Coelogyne Pandurata, 4-5 bulbs, specimens....\$25.00-35.00

249. Coelogyne Prolifera, $3-4$ bulbs........................... 7.50

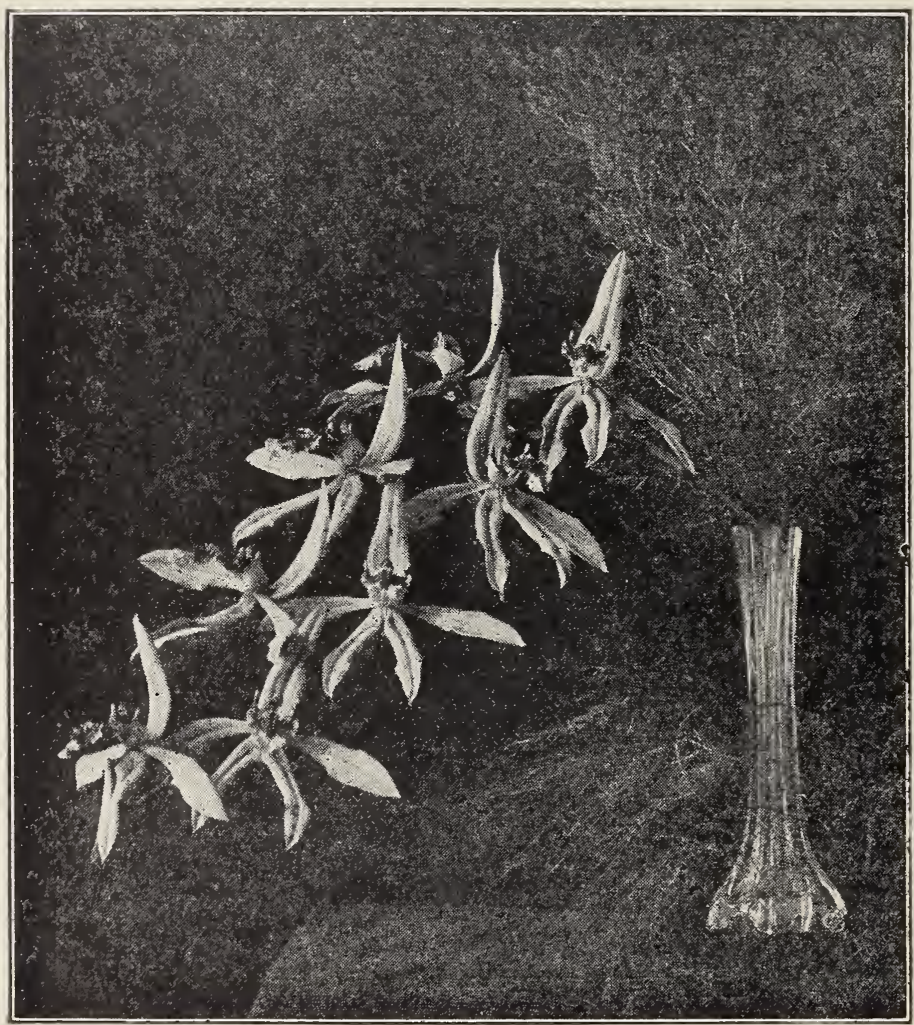

\section{Coelogyne Pandurata}

250. Coelogyne Speciosa, 5-6 bulbs........................... 3.50

251. Coelogyne Tomentosa, $5-6$ bulbs ................................ 5.00

252. Coelogyne Tomentosa, $9-10$ bulbs............................. 7.50

253. Coelogyne Sparsa-a very pretty orchid, producing 
an abundance of sprays; a dwarf growing plant, 6-10 bulbs

256. Cirrhopetalum Picturatum, 5-6 bulbs...................... 3.50

257. Cirrhopetalum Picturatum, 8-10 bulbs................... 5.00

258. Cirrhopetalum Species, $10-12$ bulbs...................... 5.00

\section{CYMBIDIUM}

One of the finest and most useful genera of orchids, both for cutting and exhibitions. The flowers are usually large and attractive. many flowers on sprays, in various colors and shades. Like the Cypripedium the flowers are of unusual lasting qualities. The plants are of easiest culture, requiring plenty of pot room, and should be potted in fibrous loam, rough peat, and sand, with good drainage, and an abundance of water during the growing season.

260. Cymbidium $\times$ Alexanderii, 3 bulbs. $\$ 25.00$

261. Cymbidium $\times$ Alexanderii, 4 bulbs. 30.00

262. Cymbidium $\times$ Alexanderii, 5 bulbs....................... 35.00

263. Cymbidium Aloefolium, 3-4 bulbs.......................... 10.00

264. Cymbidium $\times$ Beatrice, 3 bulbs........................... 20.00

265. Cymbidium $\times$ Beatrice, 4 bulbs............................... 25.00

266. Cymbidium $\times$ Butterfly, 3 bulbs........................... 20.00

267. Cymbidium $\times$ Butterfly, 4 bulbs........................... 25.00

268. Cymbidium $\times$ Corona, 4 bulbs.............................2 25.00

269. Cymbidium $\times$ Cooperii, 3 bulbs.......................... 30.00

270. Cymbidium $\times$ Doris, 3 bulbs.............................. 25.00

271. Cymbidium $\times$ Doris, 4 bulbs................................ 30.00

272. Cymbidium $\times$ Diana, 4 bulbs............................... 25.00

273. Cymbidium Eburneum, 2 bulbs............................. 10.00

274. Cymbidium Eburneum, 3 bulbs............................... 15.00

275. Cymbidium $\times$ Egret, 3 bulbs................................ 20.0 20

276. Cymbidium $\times$ Egret, 4 bulbs................................ 25.00

277. Cymbidium $\times$ Elfin, 3 bulbs................................. 25.00

278. Cymbidium $\times$ Elfin, 4 bulbs............................... 30.00

279. Cymbidium Ensifolium, 6-8 bulbs.......................... 7.50

280. Cymbidium Findlaysonianum, 4 bulbs....................... 10.00

281. Cymbidium $\times$ Gottianum, 3 bulbs............................ 25.00

282. Cymbidium $\times$ Gottianum, 4 bulbs......................... 30.00

283. Cymbidium $\times$ Geo. Woodham, 3 bulbs.................... 15.00

28. Exm

285. Cymbidium $\times$ Holfordianum, 2 bulbs....................... 20.00

286. Cymbidium $\times$ Holfordianum, 3 bulbs ..................... 30.00

287. Cymbidium $\times$ Holfordianum, 4 bulbs.................... 35.00

288. Cymbidium $\times$ Holfordianum, larger plants.....\$40.00-50.00

289. Cymbidium Insigne, 2 bulbs.................................... 10.00

290. Cymbidium Insigne, 3 bulbs...................................... 15.00

291. Cymbidium Insigne, 4 bulbs................................... 20.00

292. Cymbidium Lowianum, 3 bulbs............................. 5.00

293. Cymbidium Lowianum, 4 bulbs............................. 7.50

294. Cymbidium Lowianum, larger plants.............\$10.00-20.00

295. Cymbidium Lowianum Concolor, 3 bulbs................... 7.50

296. Cymbidium Lowianum Concolor, 4 bulbs................. 10.00 
297. Cymbidium Lowianum Concolor, larger plants..15.00-25.00

298. Cymbidium Lowianum Grandiflorum, 4 bulbs............. 20.00

299. Cymbidium Mastersii, \& butbs................................. 10.00

300 . Cymbidium $\times$ Magpie, 3 bulbs............................. 20.00

301. Cymbidium $\times$ Magpie, 4 bulbs............................. 25.00

302. Cymbidium $\times$ Moira, 3 bulbs............................. 25.00

303. Cymbidium $\times$ Moira, 4 bulbs.............................. 30.00

304. Cymbidium $\times$ Pawelsii, 3 bulbs............................ 25.00

305. Cymbidium $\times$ Pawelsii, 4 bulbs............................. 30.00

306. Cymbidium $\times$ Pawelsii, larger plants............ $\$ 40.00-50.00$

307. Cymbidium $\times$ Schlegelii, 3 bulbs........................... 20.00

308. Cymbidium $\times$ Schlegelii, 4 bulbs.............................25.00

309. Cymbidium $\times$ Schlegelii, 5 bulbs............................. 30.00

310. Cymbidium $\times$ Swallow, 3 bulbs............................ 20.00

311. Cymbidium $\times$ Swallow, 4 bulbs............................ 25.00

312. Cymbidium $\times$ Sybyl, 3 bulbs............................... 25.00

313. Cymbidium Traceyanum, 3 bulbs............................ 12.00

314. Cymbidium Traceyanum, 4 bulbs............................... 15.00

315. Cymbidium Traceyanum, 5 bulbs........................... 20.00

316. Cymbidium Traceyanum, larger plants........... $\$ 25.00-50.00$

317. Cymbidium $\times$ Veitchii, 2 bulbs.......................... 15.00

318. Cymbidium $\times$ Veitchii, 3 bulbs............................ 20.00

319. Cymbidium $\times$ Veitchii, 4 bulbs ........................... 25.00

320. Cymbidium $\times$ Veitchii, large plants...............\$35.00-40.00

321. Cymbidium $\times$ Wiganianum, 3 bulbs........................ 20.00

322. Cymbidium $\times$ Wiganianum, 4 bulbs..................... 25.00

323. Cymbidium $\times$ Wiganianum, larger plants........\$30.00-40.00

324. Cymbidium $\times$ Winnianum, 3 bulbs........................ 15.00

325. Cymbidium $\times$ Winnianum, 4 bulbs......................... 20.00

326. Cymbidium $\times$ Winnianum, larger plants.........\$25.00-30.00

327. Cymbidium $\times$ Woodhamense, 3 bulbs..................... 15.00

328. Cymbidium $\times$ Woodhamense, 4 bulbs..................... 20.00

329. Cymbidium $\times$ Woodhamense, larger plants.... $\$ 25.00-35.00$

\section{DENDROBIUM}

Most of the commercial Dendrobiums are easily cultivated, and their brightly-colored flowers are freely produced if it is borne in mind that as a general rule they require heat and abundance of moisture when growing, a season of cooler temperature, and a reasonable exposure to air and light to ripen the growth, and a drier season to produce and mature the flowers.

330. Dendrobium Acuminatum (rare) very fine, 4 bulbs....\$20.00

331. Dendrobium Albo-Sanguineum, 3 bulbs.................... 7.50

332. Dendrobium Amplum (rare), 4 bulbs....................... 5.00

333. Dendrobium Aggregatum Majus, 4-5 bulbs................ $\quad 7.50$

324. Dentrobium Ameeps, 3-4 butbs................................... 7.50

335. Dendrobium Aurantiacum, 8-10 bulbs...................... 5.00

33 6. Dendrobium $\times$ Apollo Grandiflora, 4 bulbs................ 5.00

337. Detrdrobium Bronckhardti, $-4=5$ butbs...................... 10.00

338. Dendrobium Brymerianum, 4-5 bulbs....................... $\quad 7.50$

339. Dendrobium $\times$ Brunette.

340. Dendrobium $\times$ Butterfly.

341. Dendrobium Bigibbum, 4 bulbs ............................... 5.00

342. Dendrobium Cambridgeanum, $5-6$ bulbs.................. 5.00 
343. Dendrobium Cambridgeanum, 7-8 bulbs.

10.00

344. Dendrobium $\times$ Cassiope, 4-5 bulbs.

345. Dendrobium Chrysanthum, 4-5 bulbs.........................

346. Dendrobium Chrysotoxum, 5-6 bulbs........................

347. Dendrobium Chrysotoxum, 7-8 bulbs........................

5.00

5.00

7.50

348. Dendrobium Chrysotoxum, larger plants................ 10.00

349. Dendrobium Crassinode, 4-5 bulbs............................. 5.00

350. Dendrobium Coelogyne, $4-5$ bulbs............................... 7.50

351. Dendrobium $\times$ Chessingtonense ................................

352. Dendrobium $\times$ Chrysodiscus, $4-5$ bulbs.....................

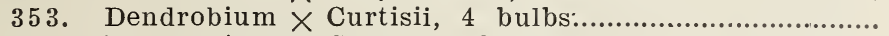

354. Dendrobium $\times$ Cybele, 4 bulbs.

355. Dendrobium Dalhousianum, 4-5 bulbs............................

356. Dendrobium Densiflorum, $4-5$ bulbs........................

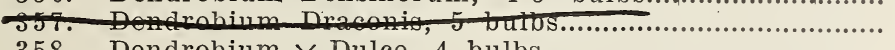

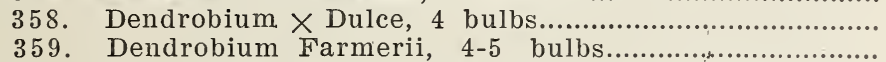

360. Dendrobium Fimbriatum oculatum, 5-6 bulbs............

361. Dendrobium Fimbriatum oculatum, 7-8 bulbs............

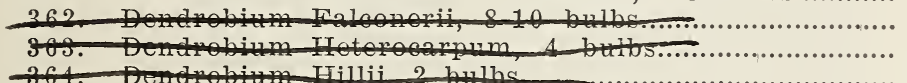

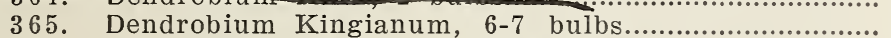

366. Dendrobium $\times$ Luna, 4 bulbs..............................

367. Dendrobium Nobile, $4-5$ bulbs.............................

368. Dendrobium Nobile, $6-7$ bulbs.................................

369. Dendrobium Nobile, 10-12 bulbs.

5.00

5.00

5.00

5.00

5.00

5.00

5.00

5.00

5.00

7.50

7.50

5.00

10.00

7.50

5.00

5.00

7.50

370. Dendrobium Nobile Amesiana, 5-6 bulbs...........................

12.50

7.50

371. Dendrobium Nobile Ashworthii, 4-5 bulbs; pure white

372. Dendrobium Nobile Burfordense, 4 bulbs...................

7.50

5.00

5.00

373. Dendrobium Nobile Childsii, 4 bulbs...........................

374. Dendrobium Nobile Cooksonii, 3-4 bulbs....................

375. Dendrobium Nobile Larz Anderson, 4-5 bulbs.............

376. Dendrobium Murrhinianum, 4 bulbs........................

377. Dendrobium Nobile Nobilium, 4 bulbs.....................

378. Dendrobium Nobile Owenianum, "4-5 bulbs.................

379. Dendrobiium Nobile Rajah, 4 bulbs...........................

380. Dendrobium Nobile Rothwellianum, 4 bulbs.................

381. Dendrobium Nobile Summitense, 4 bulbs ...................

382. Dendrobium Nobile Virginalis, " 4 bulbs........................

383. Dendrobium Regium, 4-5 bulbs..................................

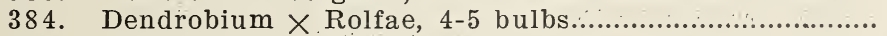

385. Dendrobium $\times$ Rubens Grandiflora, 4 bulbs...............

386. Dendrobium Suavissimum, $5-6$ bulbs.........................

387. Dendrobium Superbiens, 4 bulbs...............................

388. Dendrobium Superbum, 4-5 bulbs...........................

389. Dendrobium Superbum Dearii, white, rare, 4 bulbs...

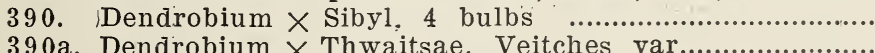

391. Dendrobium $\times$ Thelma, 4 bulbs................................

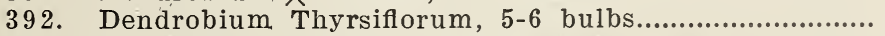

393. Dendrobium $\times$ Venus, 5 bulbs.................................

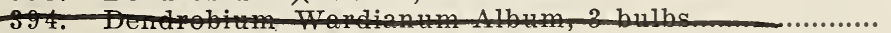

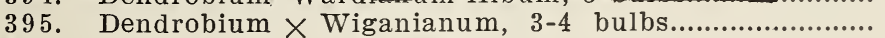

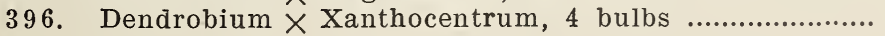

\subsection{0}

5.00

5.00

5.00

7.50

7.50

5.00

5.00

7.50 .

10.00

7.50

5.00

5.00

5.00

5.00

15.00

5.00

5.00

5.00

7.50

7.50

7.50

5.00 


\section{FPIDENDRUM}

397. Dendrobium Hybrids and fine varieties; small plants to grow on; per doz................................................... 36.0

398. Epidendrum $\times$ Boundii, 2-3 stems......................... 5.00

399. Epidendrum $\times$ Burtonii, 2 stems.......................... 3.50

400. Epidendrum Brassavolae, 4-5 bulbs....................... 5.00

401. Epidendrum Conspicuum, rare, beautiful, 5-6.......... 10.00

402. Epidendrum $\times$ Dellense, 2-3 stems........................... 7.50

403. Epidendrum $\times$ Kewensis, 2 stems.......................... 7.50

404. Epidendrum Nemorale, 5-6 bulbs.......................... 5.00

405. Epidendrum Nemorale, $7-8$ bulbs......................... 7.50

406. Epidendrum $\times$ O'Brienianum, 2-3 stems.................. 5.00

407. Epidendrum $\times$ O'Brienianum, 4-5 stems................... 7.50

408. Epidendrum $\times$ Pristes, 2 stems............................. 5.00

409. Epidendrum $\times$ Pristes, $3-4$ stems........................... 7.50

410. Epidendrum Rhizophorum, 2-3 stems...................... 3.50

411. Epidendrum Roseum, 2 stems................................. $\quad 5.00$

412. Epidendrum Virens, 5 bulbs..................................... 7.50

413. Epi-Cattleya $\times$ Nebo $1-2$ stems.............................. 7.50

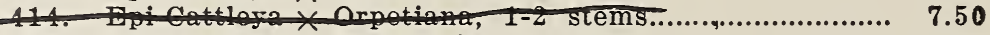

415. Epi-Cattleya $\times$ Mrs. Smith, 4 bulbs........................ 10.00

416. Epiphronitis $\times$ Veitchii, 2-3 stems.......................... 5.00

417. Eria ferruginea, $4-5$ bulbs...................................... 5.00

418. Eria species, 4 bulbs.......................................... $\quad 3.50$

419. Gomeza planifolia, $4-5$ bulbs................................ 3.50

420. Gongora Charlesworthii, 2 bulbs .......................... 5.00

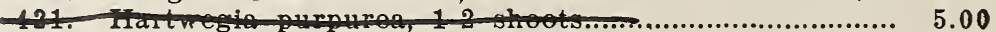

\section{LAELIA}

422. Laelia Acuminata, $5-6$ bulbs............................... 5.00

423. Laelia Acuminata, 7-9 bulbs................................. $\quad 7.50$

424. Laelia Acuminata, $10-12$ bulbs.............................. 10.00

425. Laelia Anceps, rose-purple, 5-6 bulbs........................ 3.50

426. Laelia Anceps, $7-8$ bulbs.................................... 5.00

427. Laelia Anceps, $9-10$ bulbs........................................ 7.50

428. Laelia Ánceps Alba, pure white, 4-5 bulbs.................. 10.00

429. Laelia Anceps Alba, 6-7 bulbs............................... 12.50

430. Laelia Anceps Alba, 8-10 bulbs................................ 15.00

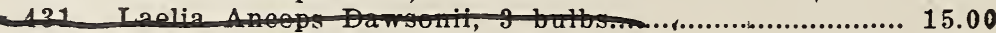

432. Laelia Anceps Grandiflora, purple, 4-5 bulbs.............. 7.50

433. Laelia Anceps Hilliana, white-rose, 4-5 bulbs............. 7.50

434. Laelia Anceps Hilliana Rosefieldense, 4-5 bulbs....... 10.00

435. Laelia Anceps Percivaliana, 4-5 bulbs......................... 7.50

436. Laelia Anceps Rosea, 4-5 bulbs................................ 7.50

437. Laelia Anceps Sanderiana, fine for cutting, 5-6 bulbs 5.00

438. Laelia Anceps Sanderiana, 7-8 bulbs....................... $\mathbf{7 . 5 0}$

439. Laelia Anceps Stella, white, fine for cutting, 5-6 bulbs 5.00

440. Laelia Anceps Stella, 7-8 bulbs................................. $\mathbf{7 . 5 0}$

41. Laelin Anceps Voitohiane, 2 bulbs ....................... 15.00

442. Laelia Anceps Williamsii, 4-5 bulbs....................... 10.00

443. Laelia Cinnabarina, 4-5 bulbs................................. 5.00

444. Laelia Crispa, 4 bulbs............................................. 5.00

445. Laelia Crispa, 5 bulbs ........................................... $\quad 7.50$

446. Laelia Crispa $\times$ Cattleya Dowiana, 4-5 bulbs............. 15.00

447. Laelia Crispilabia, 4 bulbs................................... 10.00 
448. Laelia $\times$ Dayana $\times$ Anceps Stella, 4 bulbs.............. 15.00

449. Laelia $\times$ Elegans, 4 bulbs................................... 7.50

450. Laelia $\times$ Elegans, 5 bulbs........................................... 10.00

451. Laelia $\times$ Elegans, 6 bulbs................................ 15.00

452. Laelia Gouldiana, dark purple, good for cutting,

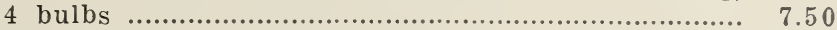

453. Laelia Gouldiana, 5 bulbs .................................... 10.00

454. Laelia Gouldiana, 6 bulbs ............................... 12.50

455. Laelia $\times$ Gravesiae, 4 bulbs................................ 5.00

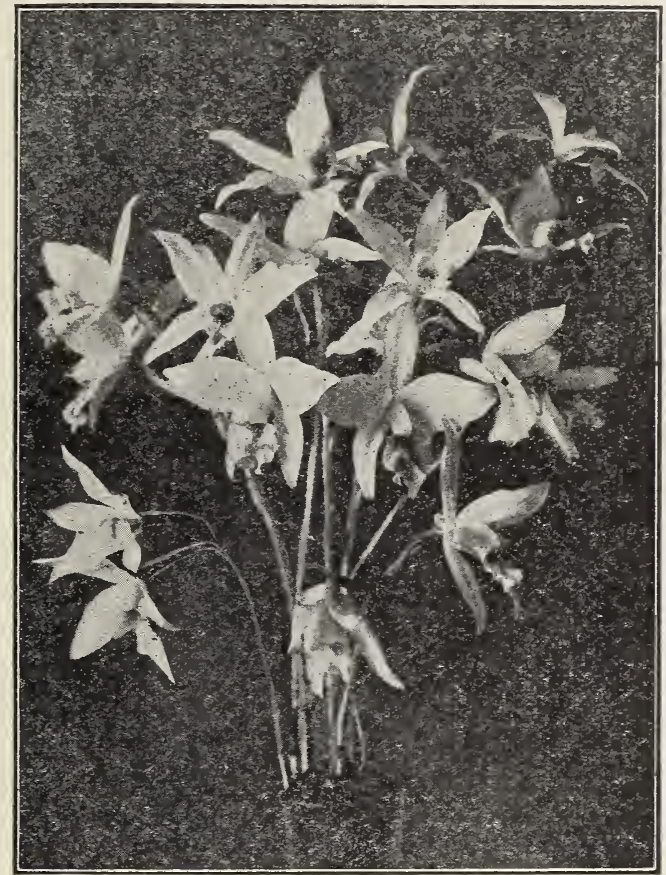

Vase of Laelia Anceps and its Albino varieties; such as Alba, Stella, Sanderiana,

Rosea, and Veitchiana

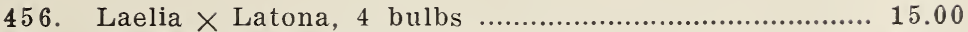

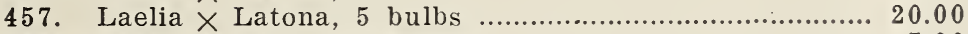

450. Mojalic 6 - \&

459. Laelia $\times$ Olivia, 4 bulbs.............................................. 15.00

460. I eli2 Dattinii A 5.00

461. Laelia Purpurata, small, 5 bulbs.......................... 5.00

462. Laelia Purpurata, strong, 5 bulbs.......................... 15.00

463. Laelia Purpurata, strong, 6 bulbs........................... 20.00

464. Laelia Superbiens, 4 bulbs................................... 10.00 
466. Laelia grandis tenebrosa, 4-5 bulbs........................ 15.00

467. Laelia $\times$ Tenebrosa $\times$ L. C. Canhamiana, 5 bulbs..... 20.00

\section{IAAELIO-CATTLEYA}

468. Laelio-Cattleya $\times$ Alphand, 4 bulbs....................... 25.00

469. Laelio-Cattleya $\times$ Aphrodite, 4 bulbs......................... 20.00

470. Laelio-Cattleya $\times$ Aphrodite, 5 bulbs.......................... 25.00

471. Laelio-Cattleya $\times$ Avon, 4 bulbs............................ 25.00

472. Laelio-Cattleya $\times$ Bardic, 4 bulbs........................... 25.00

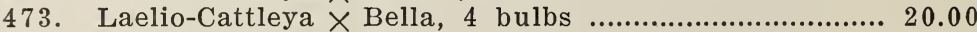

474. Laelio-Cattleya $\times$ Bella, 5 bulbs................................. 25.00

475. Laelio-Cattleya $\times$ Berth. Fournier, 4 bulbs.................2 25.00

476. Laelio-Cattleya $\times$ Bletchleyensis, 4 bulbs................. 25.00

477. Laelio-Cattleya $\times$ Brittania, 4 bulbs...................... 20.00

478. Laelio-Cattleya $\times$ Canhamiana, 3 bulbs $\ldots \ldots \ldots \ldots \ldots \ldots \ldots \ldots . \ldots 15.00$

479. Laelio-Cattleya $\times$ Canhamiana, 4 bulbs....................... 20.00

480. Laelio-Cattleya $\times$ Canhamiana, 5 bulbs....................... 25.00

481. Laelio-Cattleya $\times$ Canhamiana Rex, 4 bulbs.............. 35.00

482. Laelio-Cattleya $\times$ Calistoglossa, 4 bulbs................... 20.00

483. Laelio-Cattleya $\times$ Calistoglossa, 5 bulbs..................... 25.00

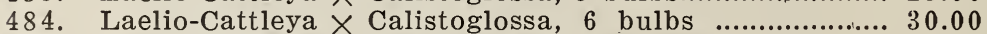

485. Laelio-Cattleya $\times$ Cowenii, 4 bulbs............................ 20.00

486. Laelio-Cattleya $\times$ Dominiana, 4 bulbs..................... 25.00

487. Laelio-Cattleya $\times$ Dominiana, 5 bulbs.......................... 35.00

488. Laelio-Cattleya $\times$ Eudora, 4 bulbs........................... 15.00

489. Laelio-Cattleya $\times$ Eudora $\times$ C. Gigas, 4 bulbs............ 18.00

490. Laelio-Cattleya $\times$ Eximia, 4 bulbs............................ 20.00

491. Laelio-Cattleya $\times$ Eximia, 5 bulbs......................... 25.00

492. Laelio-Cattleya $\times$ Fascinator, 4 bulbs...................... 10.00

493. Laelio-Cattleya $\times$ F. Boyle var. Kerchoviae, 5 bulbs.. 25.00

494. Laelio-Cattleya $\times$ G. S. Ball, 4 bulbs.......................... 15.00

495. Laelio-Cattleya $\times$ Greenwoodii, 4 bulbs...................... 20.00

496. Laelio-Cattleya $\times$ Greenwoodii, 5 bulbs................... 25.00

497. Laelio-Cattleya $\times$ Haroldiana, 4 bulbs .................... 20.00

498. Laelio-Cattleya $\times$ Hyeana, 4 bulbs................................ 20.00

499. Laelio-Cattleya $\times$ Hyeana, 5 bulbs ................................ 25.00

500. Laelio-Cattleya $\times$ Hyeana, 6 bulbs ............................... 30.00

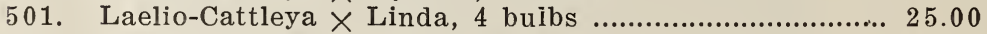

502. Laelio-Cattleya $\times$ Luminosa, 4 bulbs......................... 25.00

503. Laelio-Cattleya $\times$ Luminosa, 5 bulbs........................ 30.00

504. Laelio-Cattleya $\times$ Labiosa, 4 bulbs................................ 15.00

505. Laelio-Cattleya $\times$ Martinettii, 4 bulbs......................... 10.00

506. Laelio-Cattleya $\times$ Myra, 4 bulbs............................ 15.00

507. Laelio-Cattleya $\times$ Moonbeam, 4 bulbs....................... 25.00

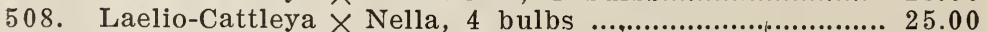

509. Laelio-Cattleya $\times$ Nysa, 4 bulbs ................................ 15.00

510. Laelio-Cattleya $\times$ Ophir, 4 bulbs ............................. 20.00

511. Laelio-Cattleya $\times$. Orpetiana, 4 bulbs........................ 10.00

512. Laelio-Cattleya $\times$ Pacavia $\times$ Gigas, 4 bulbs................. 20.00

513. Laelio-Cattleva $\times$ Veronique $\times$ Haroldiana, 4 bulbs.... 30.00

514. Lockhartia Elegans, 2-3 stems................................. 3.50

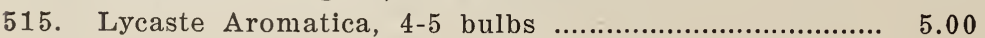


516. Lycaste Aromatica, 6-7 bulbs

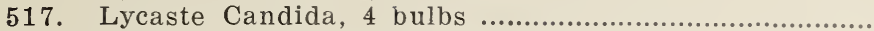

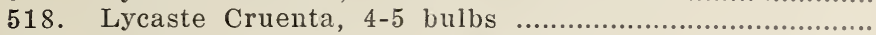

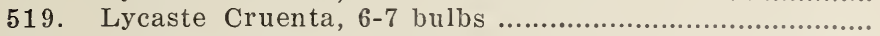

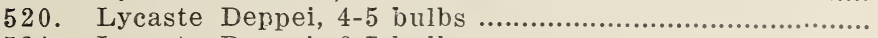

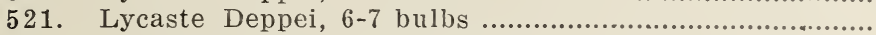

5.00

7.50

5.00

523. Lycaste Skinnerii Alba, 3 bulbs................................ 20.00

J24. Iycaste Triolor, \& bulbs.................................... 10.00

525. Mallia Species, Custa Rien, 810 leavesm........... 5.00

526. Maxillaria Hyacinthina, 4 bulbs .......................... 5.00

527. Maxillaria Incarnata, 5 bulbs ................................ 3.50

528. Maxillaria Luteo-Alba, 4 bulbs ........................... 5.00

529. Maxillaria Picta, $4-5$ bulbs ............................... 5.00

530. Maxillaria Rubescens, 5 bulbs ............................... 3.50

531. Maxillaria Tenuifolia, $41 / 2$ " pots ................................ 5.00

532. Magachlinum $\times$ falcatum, 4 bulbs ...................... 10.00

533. Miltonia, Bluntii Lubbersiana, 4-5 bulbs................. 5.00

534. Miltonia Clowesii, $4-5$ bulbs................................... 5.00

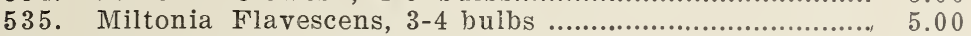

536. Miltonia Phalaenopsis, $4-5$ bulbs .......................... 7.50

537. Miltonia Roezlii, $3-4$ bulbs ............................... 5.00

538. Miltonia Vexillaria, 1 shoot ................................ 5.00

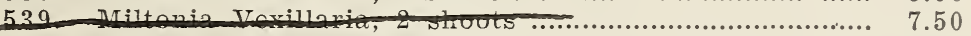

540. Neo-Benthamia gracilis, $3-4$ stems ....................... 5.00

541. Odontoglossum Citrosmum, 4-5 bulbs .................. 5.00

542. Odontoglossum Citrosmum, 6-7 bulbs........................ 7.50

543. Odontoglossum Citrosmum, 8-9 bulbs....................... 10.00

544. Odontoglossum Pulchellum, pure white, excellent for cutting, $5-6$ bulbs ............................................ 3.50

545. Odontoglossum Pelchellum, 7-8 bulbs...................... 5.00

546. Odontoglossum Pulchellum, 9-10 bulbs ........................ 7.50

547. Odontoglossum Pulchellum, larger plants........ $\$ 10.00-15.00$

548. Odontoglossum Rossii Majus, 6-8 bulbs ................. 5.00

549. Odontonia $\times$ Lairessae, 3 bulbs........................... 15.00

550. Oncidium Altissimum, 4 bulbs ............................. 5.00

551. Oncidium Ampliatum Majus, 3-4 bulbs ................... 5.00

ग5. Oneitum Anthorene, very rare,-2-3 bulbs................. 15.00

558. Oneidium Aurosum, 4-5 bulbs ............................... 5.00

554. Oncidium $\times$ Caloglossum, 4-5 leaves ........................ 10.00

555. Oncidium Cavendishīanum, 4-5 leaves ......................... 5.00

556. Oncidium Flexuosum, 1-2 shoots............................... 5.00

557. Oncidium Fuscatum, 1-2 bulbs.................................. 5.00

558 Qncidium Haprioniantm, 4 butbs ............................. 5.00

559. Oncidium Incùrvum, $4-5$ bulbs ............................ 3.50

560. Oncidium Leucochilum, $3-4$ bulbs........................... 5.00

561. Oncidium Leucochilum, 5-6 bulbs ............................ 7.50

562. Oncidium Microchilum, 5-6 bulbs ............................ 5.00

563. Oncidium Ornithorynchum, flowers small, in sprays, rose purple, good for cutting, $4-5$ bulbs..................

564. Oncidium Ornithorynchum, 6-8 bulbs ......................

565. Oncidium Ornithorynchum, 9-10 bulbs ....................... 
566. Oncidium Ornithorynchum Album, 3 bulbs

567. Oncidium Ornithorynchum, Roseum, light, pink, 3 bulbs ....

568.

Oncidium Pubes, 4-5 bulbs

10.00

569.

Oncidium Sphacelatum, 3-4 bulbs

5.00

570 .

Oncidium Sphacelatum, 5-6 bulbs

3.50

573.

Oncidium Splendidum, 5-6 bulbs

5.00

5.00

7.50

5.00

574.

Oncidium Tigrinum, 4-5 bulbs

5.00

Oncidium Wentworthianum, 5-6 bulbs .......................

575.

Oncidium Wentworthianum, 7-8 bulbs

7.50

576. Ornithidium densa, 6-7 bulbs

577. Peristeria elata, 2-3 bulbs

578. Pescatorea Cerina, 1-2 growths

Pescatorea Dayana, 1-2

5.00

580. Pescatorea Klabochorum, 1-2 growths.................... 10.00

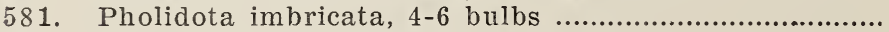

5.00

582. Plactychlinis Cobbiana, $6-8$ bulbs ......................... 5.00

583. Plactychlinis Cucumerianum, rare, $8-10$ bulbs .......... 5.00

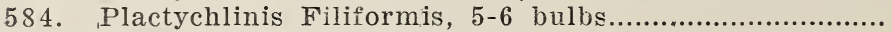

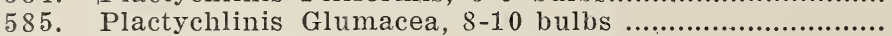

586. Plactychlinis Glumacea, $11-12$ bulbs............................

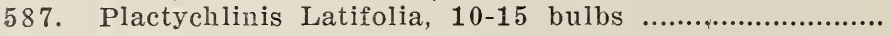

588. Pleurothallis species, 4 " pans full ...........................

589. Promenea Citrina (Zygopetalum) 6-8 bulbs .............

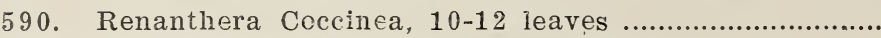

591. Renanthera Imshootiana, 8-10" high..........................

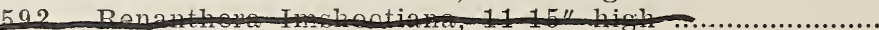

593. Saccolabium Blumei, 8 leaves ..................................

594. Saccolabium Blumei, 12 leaves ..............................

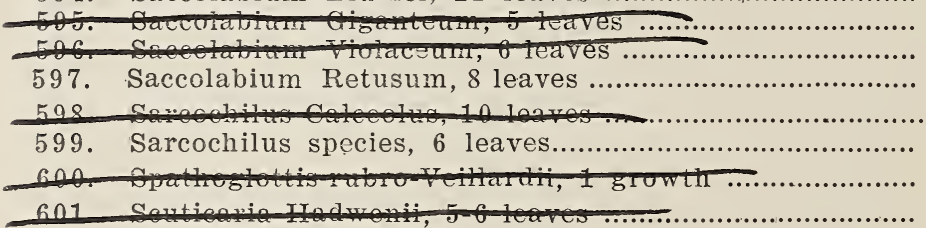

5.00

3.50

5.00

7.50

3.50

5.00

602. Sobralia Macrantha, 4-5 stems ................................

603. Sobralia Macrantha Alba, 4-5 stems .........................

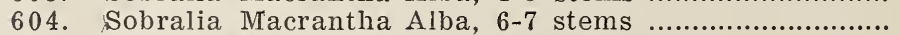

605. Sobralia Macrantha Alba, larger stems ........................

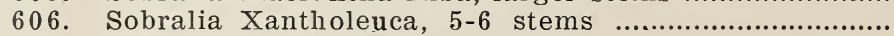

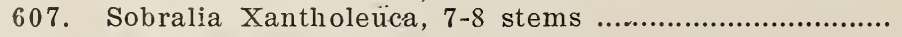

608. Schomburghia undulata, $4-5$ bulbs .............................

609. Schomburghia undulata, $6-8$ bulbs .............................

610. Stanhopea Amesiana, 4 bulbs.................................. 10.00

611. Stanhopea Insignis, 5 bulbs ............................... 5.00

612. Stanhopea Langleyensis, 3 bulbs ......................... 10.00

613. Stanhopea Reichenbachiana, 3 bulbs ....................... 10.00

614. Stanhopea Wardii, 5-6 bulbs .................................. 5.00

615. Stanhopea Wardii, var. aurea, 4 bulbs ..................... $\quad 7.50$ 
616. Thunia Marshalliana, 1 stem

617. Trichopilea Coccinea, 4-5 bulbs

618. Trichopilea Galeottiana, 4 bulbs

Thavis, $4-5$ bulbs

620. Trichopilea Tortilis, 5-6 bulbs.

VANDA

\begin{tabular}{|c|c|c|c|}
\hline & da & 11,61 & \\
\hline & Vanda & Batemanii, 8 le & \\
\hline & Vanda & Batemanii, 12 leaves & \\
\hline & $\mathrm{V}$ & 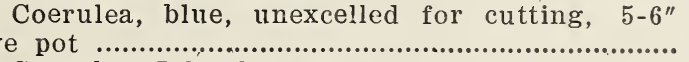 & \\
\hline 8. & Vanda & Coerulea, 7-9" above poi ................................... & \\
\hline & Vanda & Coerulea, 10-12" above pot. & \\
\hline & Vanda & Coerulea, 13-15" above pot & \\
\hline & Vanda & 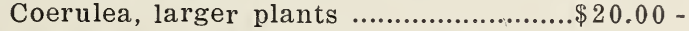 & \\
\hline 632. & Vanda & Lamellata (Boxallii), 6-8 leaves .................... & \\
\hline 633. & Vanda & Lamellata (Boxallii), 9-10 leaves................... & \\
\hline 4. & Vanda & 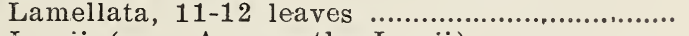 & \\
\hline & Vanda & Lowii (syn. Aracnanthe Lowii) ....................... & \\
\hline & Vanda & 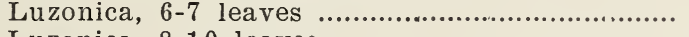 & 15 \\
\hline & Va & ica, 8-10 leaves & \\
\hline & Vanda & Luzonica, larger plants ......................\$3('.00- & \\
\hline 639 . & $\mathrm{Va}$ & $\times$ Miss Joaquim, 1 stem, 18-24" high............ & \\
\hline 640 & Vanda & 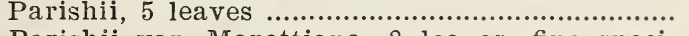 & \\
\hline & $\begin{array}{r}\text { Vanda } \\
\text { men }\end{array}$ & 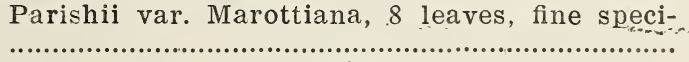 & \\
\hline 6 & Vanda & Teres, 1 stem, 15-18" high & \\
\hline 643 & Vanda & Teres Andersonii, 1 stem, 15-18" high............ & \\
\hline 64 & Vanda & Teres Aurora, 1 stem, 15-18" high................. & 7.5 \\
\hline 645 . & Vanda & 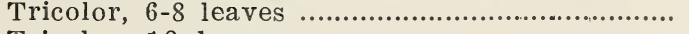 & 1 \\
\hline 6 & Vanda & n..................... & \\
\hline 6 & Vanda & Tricolor, specimen, 2 & 50.0 \\
\hline 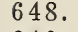 & Vanda & Tricolor, specimen, 45 leaves ........................... & 75.00 \\
\hline 64 & Vanda & Tricolor Dodgesonii, 18 leaves................ & 15 \\
\hline 65 & Vanda & Tricolor Suavis, 8-9 leaves & 15 \\
\hline 65 & $\begin{array}{l}\text { Vanda } \\
\text { Vanda }\end{array}$ & $\begin{array}{l}\text { Tricolor Suavis, } 12 \text { leaves. } \\
\text { Tricolor Suavis } 28 \text { leaves. }\end{array}$ & \\
\hline & Vanilla & $\begin{array}{l}\text { Tricolor Suavis, } 28 \text { leaves } \\
\text { planifolia, } 1-2 \text { feet high... }\end{array}$ & \\
\hline 65 & & ifolia, 1-2 feet high.. & 5.0 \\
\hline 65 & $\begin{array}{l}\text { Wa } \\
\text { Wa }\end{array}$ & iczella aromatic & 3.5 \\
\hline & & & \\
\hline & & & \\
\hline
\end{tabular}

\section{CYPRIPEDIUM (LADY SIMPPER ORCHID)}

The following list of Cypripediums is without doubt the largest and most varied collection of these plants carried by any commercial house in America. Nothing in the entire Orchid family offers such a great number of species, varieties and hybrids as do the Cypripediums. They are a constant source of interest to the cultivator. 
The flowers when cut will outlast anything else we know, and besides they are the easiest orchids of all to grow with few exceptions. In ordering please use number in left margin, and give number of list.

01 . C. $\times$ Acme (C. nitens $\times$ Bessie K. Pitcher), 3 growths $\$ 5.00$

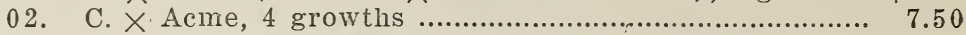

03 . C. $\times$ Actaeus (Insigne Sanderae $\times$ Leeanum), 2-3 growths .............................................................

04. C. $\times$ Acteus Langleyensis (Sanderae $\times$ Leeanum),

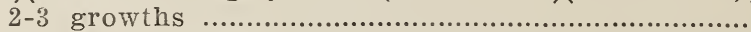

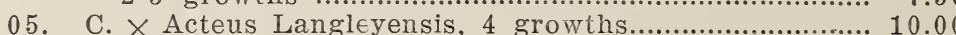

06 . C. $\times$ A. Dimmock (Druryi $\times$ Godseffianum), 2 growths 7.50

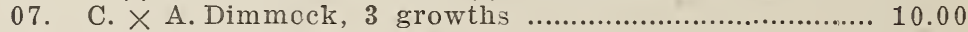

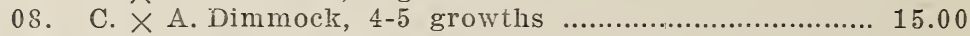

09. C. $\times$ A. de Lairess (Curtisii $\times$ Rothchildianum) 2 growths

010. C. $\times$ Alcides superbum (var.C.G.Roebling), 2 growths 15.00

011. C $\times$ Alcides superbum, 3 growths ......................... 20.00

012. C. $\times$ Alice (Stonei $\times$ Spicerianum), 3-4 growths....... 7.50

013. C. $\times$ Alcibiades superbum (Leeanum gig $\times$ Mons de Curte), 2 growths

014. C.,$\times$ Alcibiades superbum, 3 growths..................... 7.50

015. C. $\times$ Alcibiades superbum, 4-5 growths ................. 10.00

016. C.. $\times$ Archie Niel (Leeanum compactum $\times$ Harefield Hall), 2 growths

017. C. $\times$ Archie Niel, 3 growths

018. C. $\times$ Augustum (Villosum $\times$ Haynaldianum), 2 growths ...................................................... 10.00

019. C. $\times$ Augustum, 3 growths ................................ 15.00

020 . C. $\times$ Albertianum (Spicerianum $\times$ insigne var. Wallacei), 2-3 growths ...........................................

021. C. $\times$ Albertianum, 4 growths ................................

022 . C. $\times$ Albertianum Superbum, 3 growths......................

023. C. $\times$ Albertianum Superbum, 4 growths...................

024 . C. $\times$ Aureum (nitens $\times$ Spicerianum), 2-3 growths....

025. C. $\times$ Aureum Hyeanum (Spicerianum $\times$ nitens),

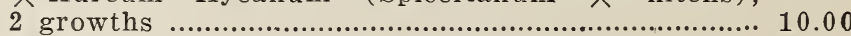

026. C. $\times$ Hyeanum, 3 growths ................................. 15.00

027. C. $\times$ Aureum Hyeanum $\times$ Men, Jerninghamii, 2 growths ........................................................... 10.00

028. C. $\times$ Aureum Hyeanum $\times$ Men. Jerninghamii, 3 growths ........................................................ 15.00

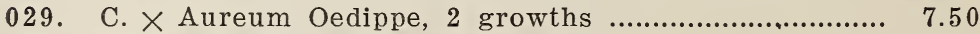

030 . C. $\times$ Aureum Oedippe, 3 growths ........................... 10.00

031. C. $\times$ Aureum Oedippe, $4-5$ growths ............................ 12.50

032. C. $\times$ Aureum surprise, $2-3$ growths........................ 10.00

033. C. $\times$ Aureum Virginale, $2-3$ growths....................... $\quad 7.50$

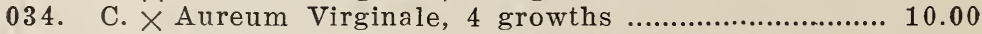

035. C. $\times$ Beatrice (Boxallii $\times$ Lowii), 2 growths ........... 5.00

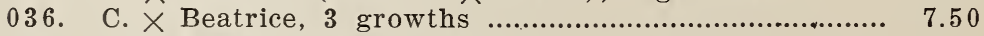

038. C. $\times$ Beekmanii $\times$ Mons de Curte, 3 growths............... 10.00

039. C. $\times$ Beryl West Point var. C. $\times$ Beekmanii $\times$ Mrs. Martyn, 2 growths 
No. Name

040. C. $\times$ Beryl West Point var., 3 growths.

041. C. $\times$ Bessie K. Pitcher (Leeanum $\times$ Villosum), 3-4 growths

C. Boxallii, 2-3 growths ...................................... 5.00

C. Boxallii, 4-5 growths ........................................... 7.50

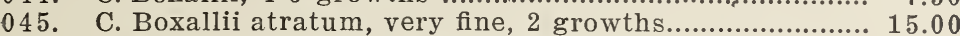

046 . C. Boxallii dilectum, 3 growths ............................... 7.50

047. C. $\times$ Boxallii $\times$ Thompsonii, 2 growths................... 10.00

048. C. $\times$ Bragaianum (hirsutissimum coerulescens $\times$ Boxallii atratum, 2 growths ................................

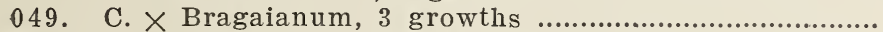

050. C. $\times$ Calypso Oakwood var. (Spicerianum), 2 growths

C. $\times$ Calypso Oakwood, $3-4$ growths ...........................

052 .

053 .

C. Charlesworthi, 3 growths

C. $\times$ Crossianum Tautzianum, 2 growths

5.00

7.50

5.00

7.50

7.50

054. C. $\times$ Curtemanii Magnificum Lows var. (Mons de Curte $\times$ Beekmanii), 2 growths............................

055. C. $\times$ Curtemanii Magnificum Lows var., 3 growths....

056

057 .

C. $X$ Dora, very fine, 2 growths...................................

C. $\times$ Dora, 3 growths

5.00

058 .

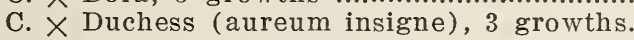

10.00

12.50

7.50

059 .

C. $\times$ Duchess, 4 growths

060 . C. $\times$ Dauthierii (Barbatum $\times$ Villosum), 3 growths...

10.00

7.50

10.00

061 .

C. $\times$ Earnestii, 2 growths ......................................

062 . C. $\times$ Fulshawense (Boxallii $\times$ Insigne Harefield Hall), 2 growths

3.50

10.00

063.

C. $\times$ Fulshawense, 3 growths .....................................

7.50

10.00

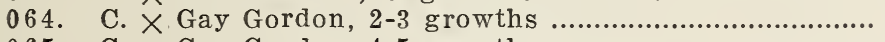

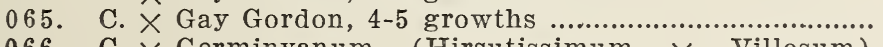

066. C. $\times$ Germinyanum (Hirsutissimum $\times$ Villosum) 2 growths

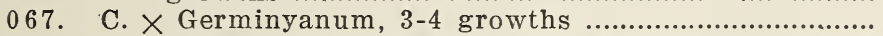

068. C. $\times$ Gigas (Lawrenceanum $\times$ Harrisianum), 2-3 grow

069. C. $\times$ Godseffianum (Boxallii $\times$ hirsutissumum), 2-3 growths

070. C. $\times$ Harrisianum (Villosum $\times$ Barbatum), 3 growths

073. C. $\times$ Harveyanum (Stonei $\times$ Leeanum), 3-4 growths

074. C. $\times$ Harveyanum, 5-6 growths

075. C. $\times$ Hera Mrs. Tautz (Nitens $\times$ Spicerianum), 2 growths

C. Insigne Burgense, 3 growths..................................

10.00

084 .

085 .

C. Insigne Chantinii. 2 growths

5.00

5.00

C. Insigne Coulsonianum, one of the finest, 2 growths 5.00 
086. C. Insigne Coulsonianum, 3 growths ....................... 7.50

087. C. Insigne Dominianum, 3-4 growths ................... 5.00

088. C. Insigne Dominianum, 5-6 growths ................... 7.50

089. C. Insigne Dominianum $\times$ Spicerianum, 2-3 growths 10.00

090. C. Insigne Fostermanii, 3 growths ........................ 5.00

091. C. Insigne Gravesianum, 3 growths ........................ 3.50

092. C. Insigne Harefield Hall, 2 growths .................... 5.00

093. C. Insigne Harefield Hall, 3 growths ..................... $\quad 7.50$

094. C. Insigne Harefield Hall, 4 growths ................... 10.00

095. C. Insigne Harefield Hall, larger plants...........15.00-25.00

096. C. Insigne Harefield Hall $\times$ Spicerianum, 3-4 growths 10.00

097. C. Insigne Harelield Hall $\times$ Spicerianum, 5-6 growths 12.50

098. C. Insigne Hurrellianum, 3 growths ........................ 3.50

099. C. Insigne Hurrellianum, 4 growths ....................... 5.00

0100. C. Insigne Hurrellianum, 5 growths ........................ 7.50

0101. C. Insigne Hurrellianum, larger plants ..........10.00-15.00

0102. C. Insigne Julius Roehrs, 2 growths ....................... 10.00

0103. C. Insigne Lagerae, 2 growths ............................. 5.00

0104. C. Insigne Lagerae, 3 growths ............................. 7.50

0105. C. Insigne Lagerae, 4 growths ................................. 10.00

0106. C. Insigne Lindeniae, 3-4 growths .......................... 5.00

0107. C. Insigne Lutea alba, 3 growths ........................... 3.50

0108. C. Insigne Monarch $\times$ Nitens, 2-3 growths ............. 10.00

0109. C. Insigne Aureola, yellow variety, 3 growths ........... 3.50

0110. C. Insigne Aureola, yellow variety, 4 growths ........... 5.00

0111. C. Insigne Aureola, yellow variety, 5 growths ............ 7.50

0112. C. Insigne Balliae, yellow variety, 3 growths ........... 5.00

0113. C. Insigne Balliae, superbum, 3 growths ................. 7.50

0114. C. Insigne Brightness, yellow variety, 2-3 growths.... 5.00

0115. C. Insigne Brightness, yellow variety, 4-5 growths... 7.50

0116. C. Insigne Brightness, larger pants ..............10.00-12.50

0117. C. Insigne Chantini Lindeni, yellow var., 2-3 growths $\mathbf{7 . 5 0}$

0118. C. Insigne Dorothy, yellow var., 3 growths.............. 5.00

0119. C. Insigne Laura Kimball, yellow var., 2-3 growths... $\mathbf{7 . 5 0}$

0120. C. Insigne Laura Kimball, yellow var., 4-5 growths 10.00

0121. C. Insigne Lucianiae, yellow var., 2-3 growths........... 7.50

0122. C. Insigne Sanderae, yellow var., 3 growths............... 5.00

0123. C. Insigne Sanderae, yellow var., 4 growths............... 6.00

0124. C. Insigne Sanderae, yellow var., 5 growths............. $\quad 7.50$

0125. C. Insigne Sanderae, yellow var., 6 growths.............. 10.00

0126. C. Insigne Sanderae, yellow var., larger plants $15.00-25.00$

0127. C. Insigne Sanderae $\times$ Chantinii Lindenii, 2 growths 10.00

0128. C. Insigne Westgatense, yellow var., 3 growths......... 5.00

0129. C. Insigne Westgatense, yellow var., 3-4 growths....... $\quad 5.00$

0130 . C. Insigne Westgatense, yellow var., 5-6 growths....... 7.50

01.31. C. Insigne Xanthinum, yellow var., 4-5 growths....... 5.00

0132. C. $\times$ Josenhianum, 3 growths .............................. 5.00

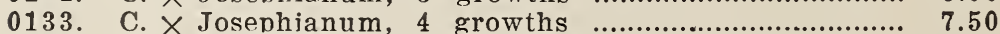

0134. C. $\times$ Lathamianum (Spicerianum $\times$ Villosum), 3-4 growths

0135. C. $\times$ Lathamianum Negleys variety, a superior form of the preceding, 3-4 growths

0136. C. $\times$ Leeanum (Insigne $\times$ Spicerianum), 4-5 growths $\mathbf{5 . 0 0}$ 
C. $\times$ Leeanum Clinkaberryanum, this grand Cypripedium stands unrivaled today for its charming beauty and distinctiveness, 3 growths

c. $\times$ Mahlerae (Rothschildianum $\times$ Lawrenceanum) 1 growth

0158.

C. $\times$ Mahlerae, 2 growths

0159. C. $\times$ Minos Lowii (Spicerianum $\times$ Arthurianum pul( chellum), 3 growths

0160.

C. $\times$ Minos Lowil, 4 growths

C. $\times$ Minos Youngii (Arthurianum $\times$ Spicerianum) 2 growths

0162 .

C. $\times$ Mrs. Chas. Canham, 2-3 growths.

0168. C. Nitens (villosum $\times$ insigne Maulei), 3 growths....

0169. C. $\times$ Nitens Gravesianum, 3-4 growths......................

0170. C. $\times$ Osbornei (Harrisianum Superbum $\times$ Spiceri' anum, 2-3 growths

0171. C. $\times$ Premier (Beechense $\times$ Rothschildianum) 2-3 growths

0172. IC. $\times$ Premier, 4 growths .........................................

0173. C. $\times$ Prewetii (Harrisianum sup. $\times$ Lawrenceanum) 2-3 growths

0174. C. $\times$ Priapus (Phillippinense $\times$ villosum), 3 growths

0176. C. $\times$ Rothschildiano-superbiens, 2 growths .............. 10.00

0177 . C. $\times$ Rothschildiano-superbiens, 3 growths $\ldots . . . \ldots \ldots \ldots . . . .115 .00$

0178 . C. $\times$ Rothschildiano-tonsum, 2 growths ................ 10.00 


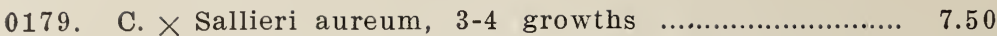

0180 . C. $\times$ Sallieri nigro maculatum, $2-3$ growths $\ldots \ldots \ldots \ldots . . .6 \%$

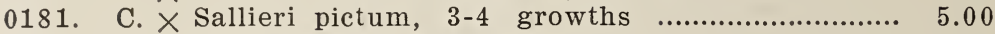

0182. C. $\times$ Platypetalum, 3 growths ........................... 5.00

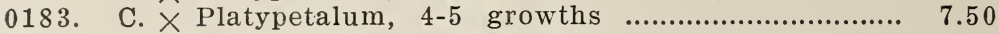

0184. C. $\times$ Schleseringerianum (Roxallii $\times$ insigne Maulei), $2-3$ growths ..................................................... 5.00

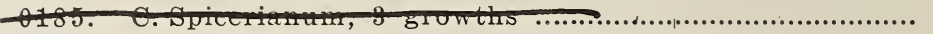

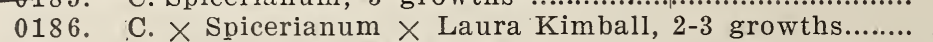

0188. C. $\times$ Tityus (Spicerianum $\times$ Cenanthum superbum.)

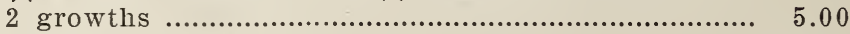

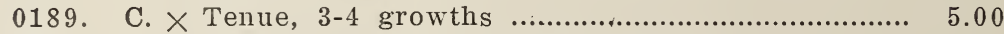

0190. C. $\times$ Tonso-Arthurianum, 2 growths ...................... 5.00

0191. C. $\times$ Tonso-Villosum (Tonsum $\times$ villosum),

0192. C. $\times$ Tonso-Villosum, $3-4$ growths ....................... 7.50

0193 . C. $\times$ Thompsonii (Spicerianum villosum), 2-3 growths 7.50

0194 . C. $\times$ Transval (Chamberlainianum $\times$ Rothschildianum), 2 growths ........................................... 10.00

0195. C. $\times$ Transvaal, 3 growths ................................... 15.00

0196. C. $\times$ Triumphans (nitens $\times$ cenanthum), 2-3 growths 10.00

0197. C. $\times$ Van Dyke (Hirsutissimum $\times$ Mons de Curte), 2 growths .................................................... 15.00

0198. C. $\times$ Ville de Paris (Insigne Harefield Hall $\times$ Nitens),

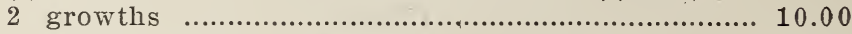

0199. C. $\times$ Ville de Paris, 3 growths .............................. 12.50

0200 . C. Villosum, $2-3$ growths ........................................... $\quad 5.00$

0201. C. $\times$. William McKinley, 2 growths ........................ 5.00

0203 . C. $\times$ William McKinley, 3 growths ...................... 7.50

0204 . C. W. W. Lunt, (Boxallii $\times$ insigne), 2-3 growths..... 5.00

0205 . C. W. W. Lunt, 4-5 growths............................... 7.50

\section{CYPRIPEDIUM-Continued}

\section{Selenipedium Section}

These are so distinct in regard to habit of the plants, colors and shapes of the flowers, that they really form a separate group and we have listed them below, apart from the others: Many of them have long, ribbon-like petals, and all produce a number of flowers on tall scapes, one after another. It is thus possible to have these plants in flower for: a long time, for as soon as one flower drops, another takes its place.

The colors of many are white, or nearly so", others rose pink to purple, while some are green, suffused with yellow and purple. They are of easiest culture and form handsome and stately subjects in the greenhouse or conservatory when in flower.
0206 .
C. $\times$ Albo-purpureum (Schlimii $\times$ Dominianum),

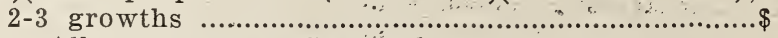

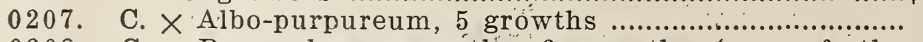
0208 . C. $\times$ Brysa, large growths, 3 growths (one of the 5.00 finest)

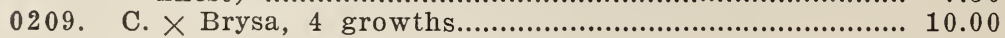




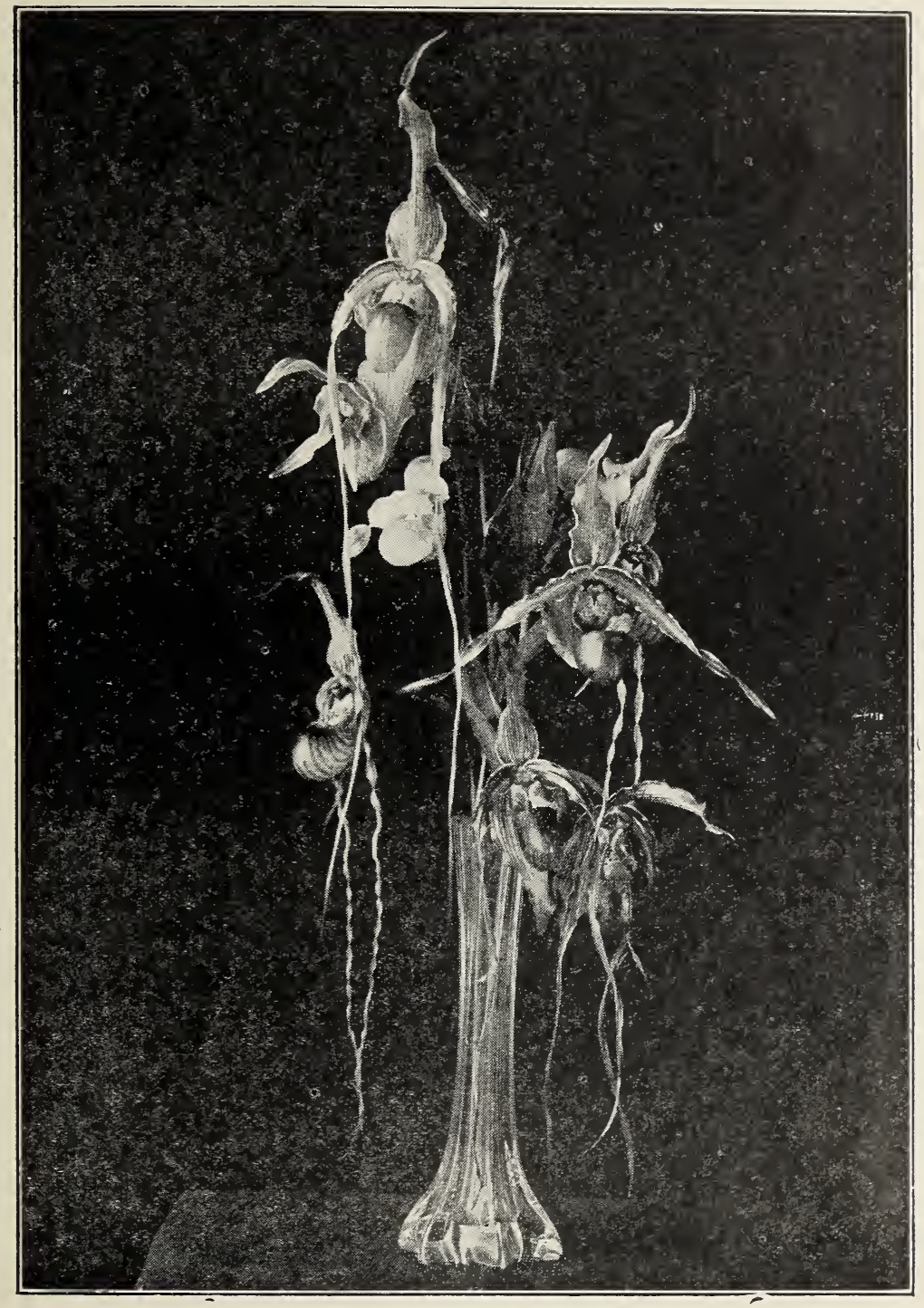

Cut flowers of Selenipediums in variety. Note the one at the top with its very long ribbon-like petals. It is S. Nitidissima 
0210. C. $\times$ Brownil (Leucorrhodum $\times$ longifolium, var. Magniflorum ), 2-3 growths ....................................

211. C. $\times$ Brownii leucoglossum, superior form of the preceding, 3 growths

0212.

C. $\times$ Cunia (longifolium

0213 $\times$ Conchiferum (Caricinum $\times$ longifolium, var. Hartwegii), 3 growths.

0214. C. $\times$ Calurum (longifolium $\times$ Sedenii), 3 growths......

0215.

0216.

0217.

C. $\times$ Calurum Rougierii, 2 growths

C. $\times$ Cardinale, 2-3 growths

C. $\times$ Cleola (reticulatum $\times$ Shlimii albiflorum), 3 growths...

0218. C. $\times$ Dominianum (Caricinum $\times$ Caudatum), 4-5 growths

0219. C. $\times$ Eastonii (Longforium $\times$ Lindleyanum), 2-3 growths

0220 .

C. $\times$ Grande (caudatum $\times$ Longifolium), 2-3 growths

0221.

C. $\times$ Leucorrhodum (Reezlii $\times$ Schlimii Albiflorum) 2 growths

0222.

C. $\times$ Lindleyanum, 2-3 growths

0224 .

C. $\times$ Lindleyanum $\times$ C. Leucorrhodum, 2 growths....

C. $\times$ Magniflorum, 2 growths

0225 . C. $\times$ Macrochilum giganteum (Grande $\times$ Uropedium Lindenii), 2 growths

0226. C. $\times$ Mrs. W. A. Roebling (caudatum $\times$ Leucorrhodum ), 2-3 growths

0227. C. $\times$ Nitidissimum (Caudatum $\times$ Warscewiezii) one of the finest of all. The ribbon-like petals measure 15" to $20^{\prime \prime}$ in length. As good as C. Caudatum, and better because it is easy to grow; 2 growths

0228.

C. $\times$ Nitidissimum, 3 growths ...............................

0229 .

C. $\times$ Nitidissimum, 4 growths

0.230 .

C. $\times$ Penelaus (calurum $\times$ caudatum), 2 growths....

0231.

C. $\times$ Perseus (Sedenii porphyreuin $\times$ Lindleyanum) 2 growths

0232.

C. Roezlii, 2 growths

0234 . C. $\times$ Schroederae (Caudatum ; Sedenii), 3-4 growths

0235 . C. $\times$ Schroederae splendens, 3 growths.......................

0236. C. $\times$ Sedenii candidulum (longifolium $\times$ Schlimii), 2-3 growths

0237. C. $\times$ Tenellum (longifolium magnificum $\times$ Schlimii), 2 growths

0238. C. $\times$ Titanum (Lindleyanum $\times$ longifolium), very large, 2 growths

0239. C. $\times$ Uranus (Lindleyanum $\times$ grande), 2 growths... 15.00

0240. C. $\times$ Uranus, $3-4$ growths, very large.................... 25.00

0241. C. $\times$ Urgandae, Graves var. (Lindleyanum $\times$ Roezlii roseum!), a large, magnificent growing Cypripedium, 3 growths

0242 . C. $\times$ Urgandae, Graves var., 4 growths, very large.... 20.00 


\section{COLLECTIONS-SPECIAL OFFER}

Special offers to amateurs and new beginners. For the conveni ence of these we have arranged the following collections of Orchids consisting of the very best kinds, our selection:

Collection No. $1-50$ plants in 50 varieties for.

Collection No. 2-25 plants in 25 varieties for................ 75.00

Collection No. $3-12$ plants in 12 varieties for.................. 37.50

Collection No. 4 - 6 plants in 6 varieties for.................. 18.75 cluded.

In all of the above collections some Cypripediums will be in-

\section{COLLECTION OF CYPRIPEDIUMS-OUR SELECTION}

Collection No. $5-25$ plants in 25 varieties for...............\$75.00

Collection No. $6-12$ plants in 12 varieties for................. 37.50

Collection No. 7 - 6 plants in 6 varieties for..................... 18.75

Collection No. 8-(Selenipediums) 6 plants in 6 varieties... 18.00

Collection No. $9-12$ plants in 12 varieties for................. 30.00

Collection No.10-Botanical orchids, mostly unnamed, our selection, 6 plants for.................................... 12.08

\section{NATIVE, HARDY ORCHIDS}

For moist shady places in the garden or woodland. These we can supply only during spring and fall.

$\begin{array}{crr}\text { Each } & \text { Doz. } & 100 \\ \text { Calopogon pulchellum, desirable for bog.......25c } & \$ 2.50 & \$ 20.00 \\ \text { Cypripedium Acaule, red, ladies' slipper.......35 } & 3.50 & 25.00 \\ \text { Cypripedium Parviflorum, ......................35c } & 3.50 & 25.00 \\ \text { Cypridedium Pubescens, yellow, fine...........35c } & 3.50 & 25.00 \\ \text { Cypripedium Spectabile, large, white and } & & \\ \text { pink ............................................40c } & 4.00 & 30.00\end{array}$

\section{MATERIALS AND SUPPLIES FOR ORCHID CULTURE} Square Orchid Baskets

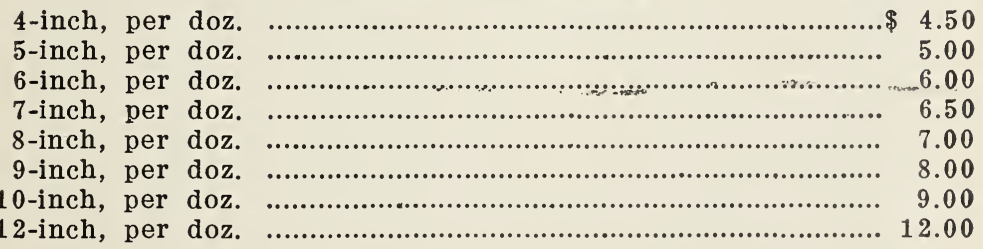

Selected orchid peat, per sack........................................... 3.50

Selected orchid peat, chopped, ready for use, per sack.......... 6.00

Live sphagnum moss for orchids, per sack........................... 3.50

Live sphagnum moss, picked, per sack............................. $\quad 6.00$

Charcoal for orchids, per sack.......................................... 3.00

Leafsoil for orchids, per sack......................................... 5.00

Peatsoil, for general use, per sack................................... 2.50

Celluloid labels with metal eyelets, $21 / 4 \times 11-16$, per $100 \ldots \ldots \ldots \ldots . . .22 .00$

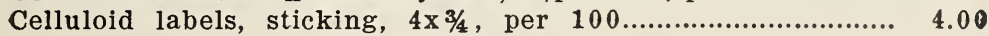


Canadian unleached hardwood ashes. An excellent fertilizer for lawns and vegetables in general:

$100 \mathrm{lb}$. bag ............................................................. $\$$

500 lbs.

Per ton

\section{INSECTICIDLS FOR ORCHIIS}

Aphine-very effective as a spray against white, black and green fly, thrips, and scale, etc.,-pint, $65 \mathrm{c}$; qt., $\$ 1.00$; gal., $\$ 3.00$.

Nicoticide-The most effective insecticide we know of for thrips, green fly, red spiders, etc.; may be used for spraying or fumigation. 8-1b. can, $\$ 15.00 ; 4-1 b$. can, $\$ 7.75 ; 2-1 b$. can, $\$ 3.90$. Fumigators for above, complete, $\$ 1.00$

\section{BOOKS ON ORCHIDS}

During and since the war, books on orchids have not been available, except a few copies We have a copy of each of the following listed below which we offer subject to being unsold when ordering. We may be able to secure others from time to time, but we cannot tell at present what they will be. Hence we advise prospective customers for books on orchids to write us and if we have any on hand we will be glad to state the kind of books we have and prices of same.

"Manual of Orchidaceous Plants," by James Veitch \& Sons. Freely illustrated with woodcuts and maps. 2 vols., cloth 8 vo. One set only, price $\$ 75.00$. The above has always been and is now a classic on orchids.

"Orchids, The Royal Family of Plants," with illustrations from nature, by Harriet Stewart Miner. A splendid work on orchids, with 24 colored plates, measuring $10^{\prime \prime} \times 12 \frac{1}{2}$ ". 90 pages, one copy only, $\$ 25.00$.

"The Orchid Stud Book," by Robt. Allen Rolfe and Charles Chamberlain Hurst. An enumeration of Hybrid Orchids of artificial origin, with their parents, raisers, date of first flowering, etc., with an historical introduction and a chapter on hybridizing and raising orchids from seed, $\$ 7.50$.

"The Orchid-Grower's Manual," by B. S. Williams. 8vo, gilt edge, cloth, 300 illustrations. Descriptions of all kinds of orchids and general cultivation. One copy only, price $\$ 20.00$.

"Fertilization of Orchids," by Charles Darwin. Illustrated. One copy only, price, $\$ 5.00$.

Yours respectfully,

LAGER \& HURRELL. 


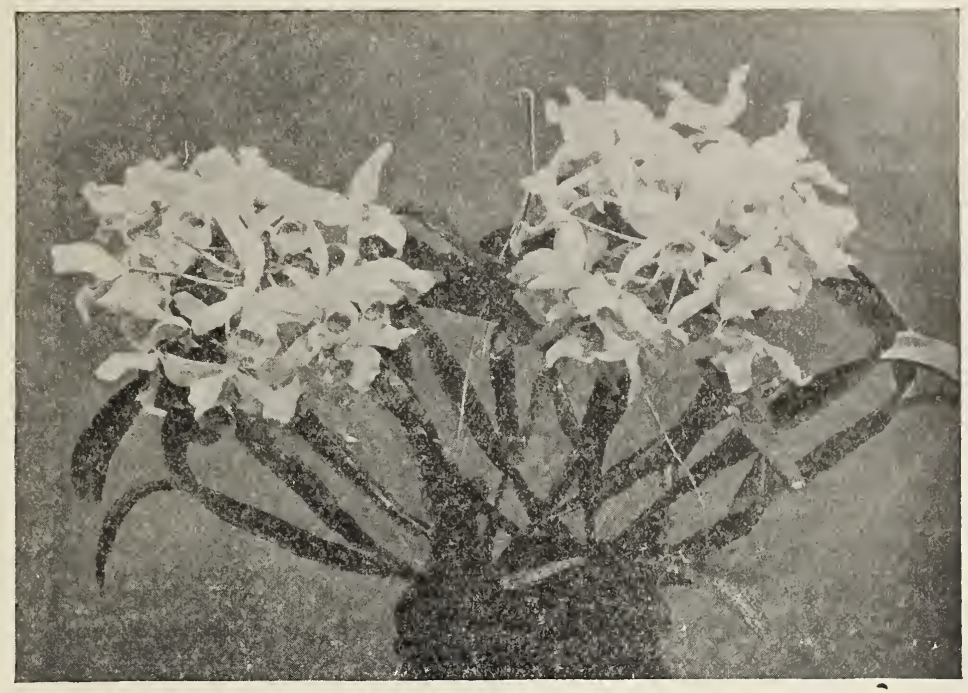

Cattleya Skinnerii.- See page 7

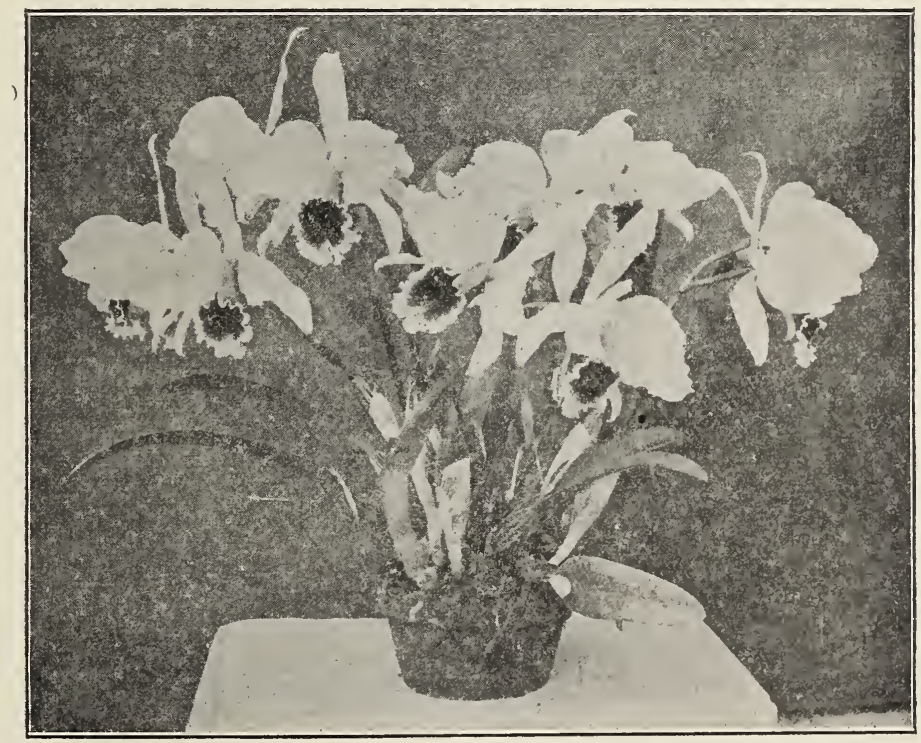

Cattleya Percivaliana-Seo pages $6-7$ 

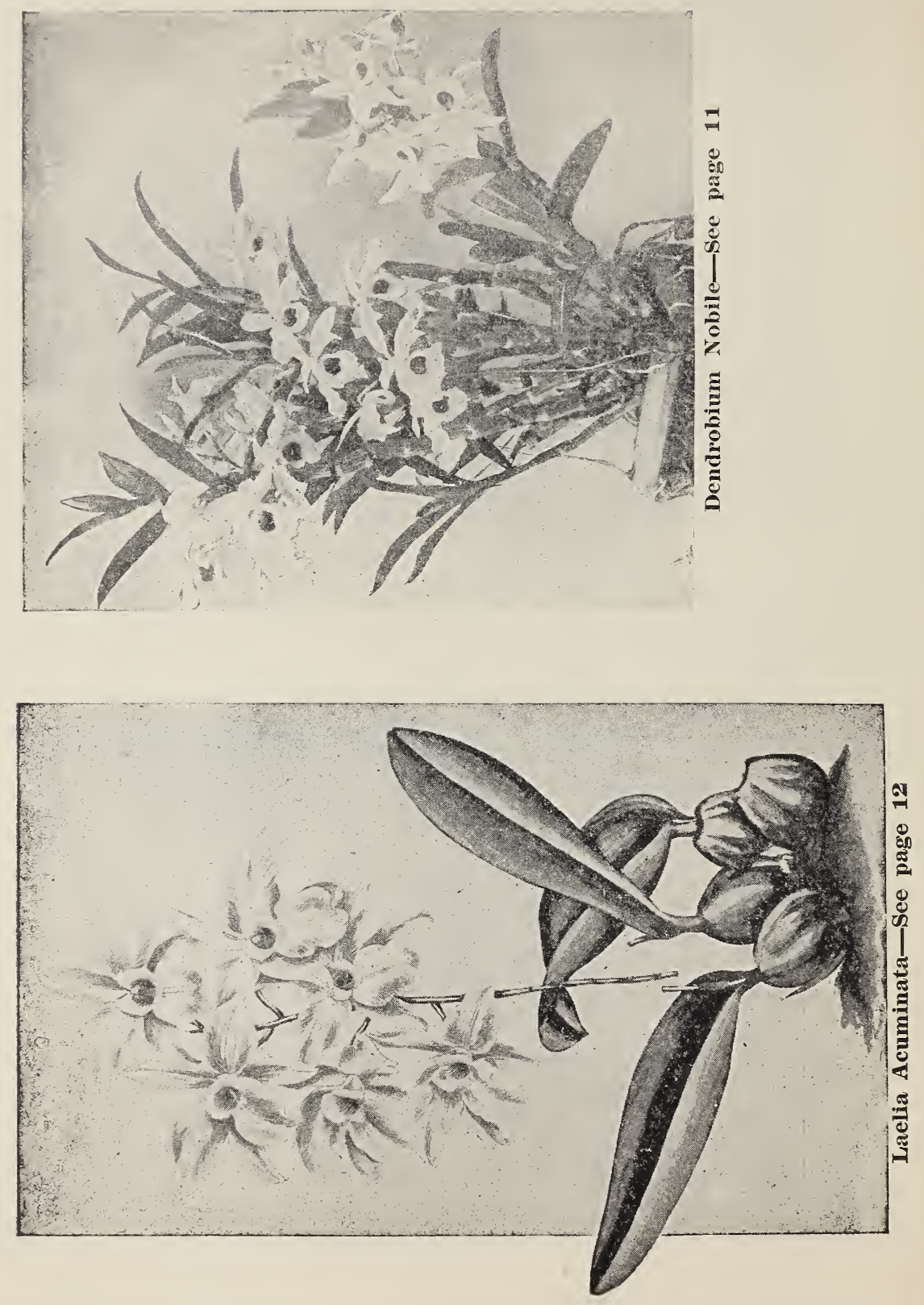


\section{ORDER SHEET

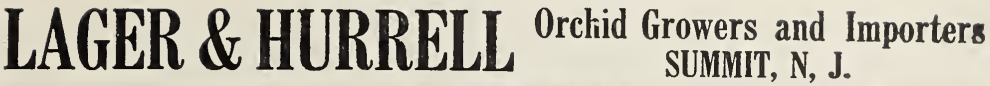

\section{IN ORDERING USE THIS SHEET}

Please give Explicit Shipping Directions with Every Order THE PRICES IN THIS LIST CANCEL ALL

Dear Sirs :

$$
\text { PREVIOUS QUOTATIONS }
$$

Fnclosed find Post Office Money Order, Amount, $\$$

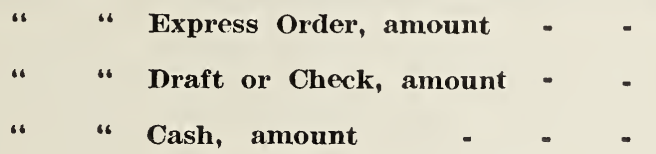

to the address or

For which send the following by (freight or express),

Name

Post Office.

.County

State Express Office or

Railroad Station

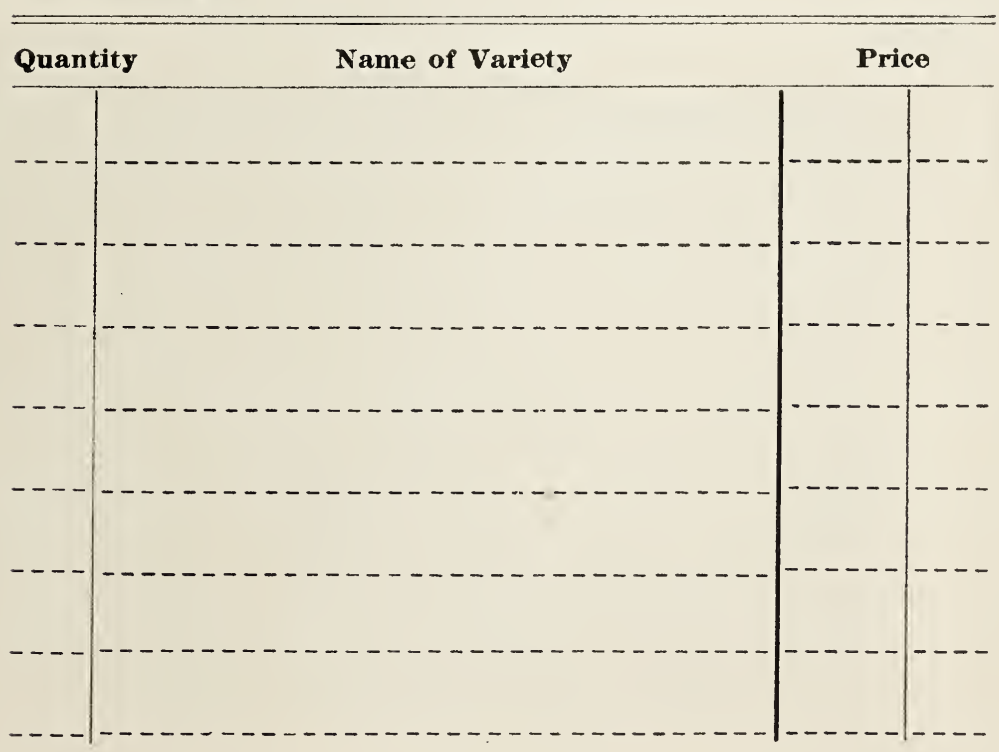




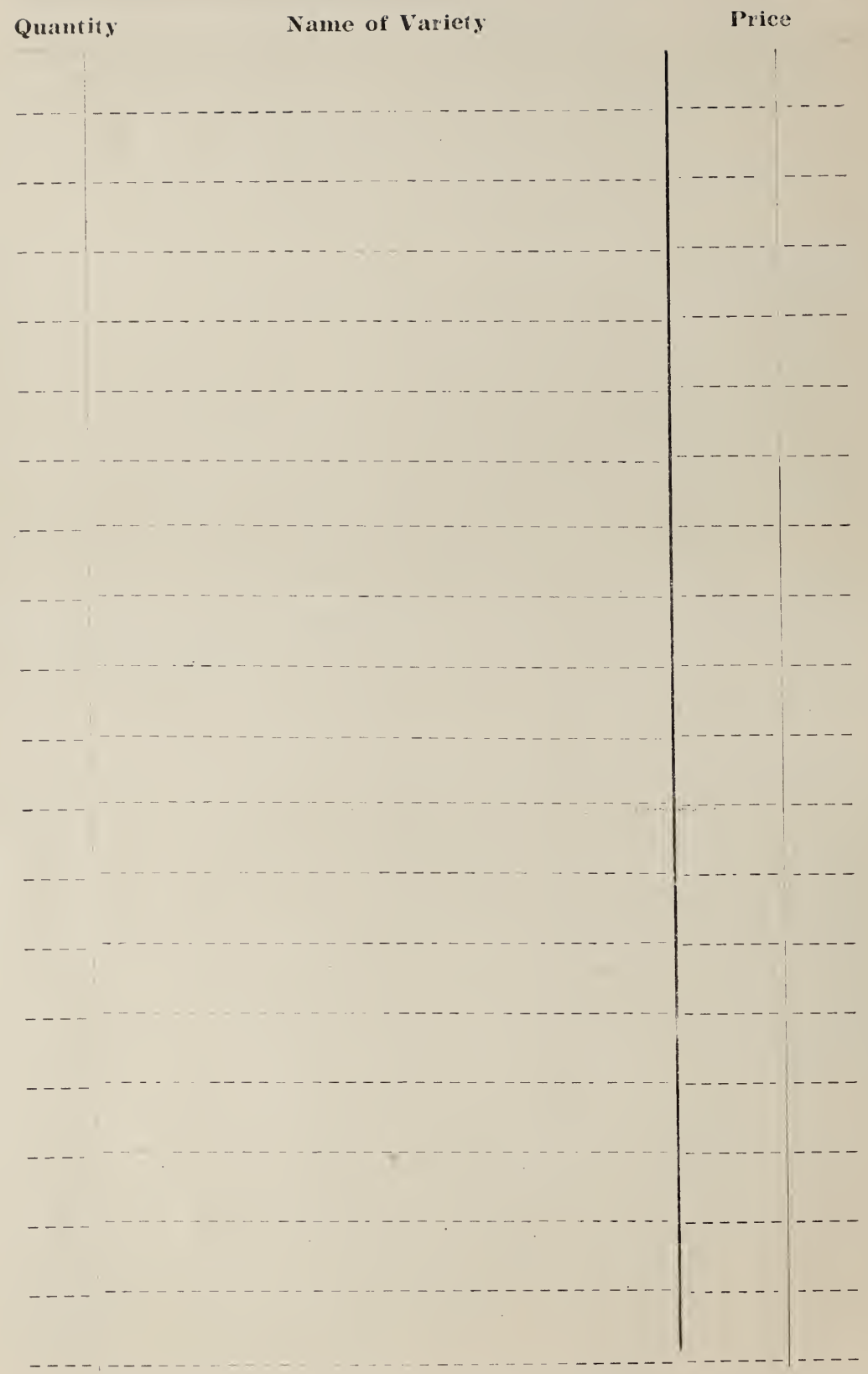




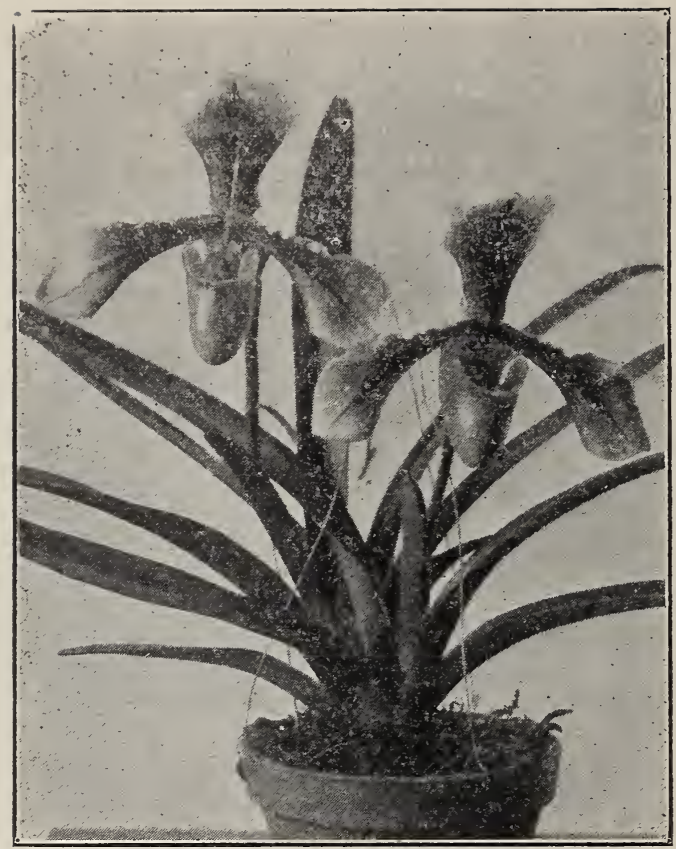

Cypripedium $\times$ Alcıdes superbum, var. C. G. Roebling-See page 18

\section{HOW TO KEEP CUT ORCHIDS}

Orchids as a whole keep longer cut or on the plants than most other flowers. This is due to the presence of mucilage in the flowers. The question, however, is very often asked, how can I keep my orchids after they are cut?

In answer to this, the following will probably be useful. If you have orchids in your greenhouse cut the flowers in the morning or late in the evening. Never cut them when the sun is full on the glass. Place them in cold water in any place free from gas and where the temperature does not exceed $60^{\circ}$. Leave the flowers in this position for several hours before using them. Whenever practical use an earthenware vase instead of glass or metal. When the flowers are used on the table, and they have served the purpose remove them and place them as indicated above in an earthenware vase, and in a temperature of minimum $56^{\circ}$, maximum $60^{\circ}$. The next time after the flowers have been used cut off the lower part of the stem 1-16 of an inch, and replace in water. Never subject your flowers to a low temperature. They are tropical and cannot stand cold with impunity. If you follow these instructions you will be able to enjoy your orchids much longer than in any other way. LAGER \& HURRELL - - SUMMIT, NEW JERSEY 